\title{
Differential Loss of Neuromuscular Connections According to Activity Level and Spinal Position of Neonatal Rabbit Soleus Motor Neurons
}

\author{
Edward M. Callaway, ${ }^{a}$ James M. Soha, ${ }^{b}$ and David C. Van Essen \\ Division of Biology, California Institute of Technology, Pasadena, California 91125
}

\begin{abstract}
We have tested whether the ability of synapses to compete for occupancy of endplates during neuromuscular synapse elimination is affected by differences in the spinal position or in the activity level of the parent motor neuron. To test the role of spinal position, the relative sizes of motor units for motor neurons from middle and extreme (rostral/caudal) positions in the rabbit soleus motor pool were determined at 3 postnatal ages: 4-5 d ("early" ages, when the soleus is heavily polyinnervated), 8-9 d ("intermediate"), and 11$15 \mathrm{~d}$ ("late," when the soleus has just reached the singly innervated state). Average motor unit sizes from extreme ventral roots were similar to those from middle ventral roots in early-aged soleus muscles but were significantly smaller (by 18-27\%) for both intermediate and late muscles. Thus, motor neurons from extreme positions evidently compete less effectively for retention of synapses than those from middle positions.
\end{abstract}

To test the role of differential activity, inactive and active synapses were pitted directly against one another by implanting Silastic plugs laden with tetrodotoxin (TTX) into one of the spinal nerves containing a minority of the soleus motor axons. Differential activity was maintained during a period of extensive synapse loss, from the time of the implant at day 4 or 5 until the intermediate age (day 8-9). Motor unit twitch tensions were subsequently measured to determine the relative number of synapses retained by individual active and inactive motor neurons. The inactivated motor units were on average significantly larger (by more than $50 \%$ ) than the corresponding group from normal and control-implanted animals. The abnormally large size of inactivated motor units persisted in animals allowed to recover from the TTX block and examined after multiple innervation had disappeared. Hence, the effect of the TTX block cannot be attributed to a simple slowing of synapse elimination specifically among the inactive motor neurons. We conclude that complete presynaptic inactivity improves the chances of survival relative to that for normal activity during synapse elimination in the neonatal rabbit soleus muscle. This difference in competitive

\footnotetext{
Received June 30, 1988; revised Oct. 10, 1988; accepted Oct. 26, 1988.

This work was supported by NSF Grant BNS 8408213 and by NIH Training Grants T32 GM07737 to E.M.C. and T32 GM07616 to J.M.S. We thank Richard Ribchester and Bill Betz for comments on the manuscript.

Correspondence should be addressed to Edward M. Callaway, Laboratory of Neurobiology, Box 138, Rockefeller University, 1230 York Avenue, New York, NY 10021.

a Present address: Laboratory of Neurobiology, Box 138, Rockefeller University, 1230 York Avenue, New York, NY 10021.

'Present address: E. K. Shriver Center, 200 Trapelo Road, Waltham, MA 02254.

Copyright (C) 1989 Society for Neuroscience $0270-6474 / 89 / 051806-18 \$ 02.00 / 0$
}

ability may contribute to the development of an important characteristic of adult muscles, the correlation between motor unit size and recruitment threshold.

In newborn mammalian muscle, each muscle fiber is innervated at a single endplate by several different motor neurons. During the first weeks of postnatal development the redundant synapses are lost, leaving each fiber innervated by exactly one motor neuron (Redfern, 1970; Brown et al., 1976). Several observations indicate that the individual terminals at a polyinnervated endplate compete with one another for occupancy of the endplate. After partial denervation at birth, the remaining neurons lose fewer synapses than normal, indicating that motor neurons do not simply lose a predetermined number of synapses independent of interactions with other terminals (Brown et al., 1976; Thompson and Jansen, 1977; Betz et al., 1980; Fladby and Jansen, 1987). Competition is also a plausible hypothesis for cxplaining how single innervation develops without the appearance of denervated endplates.

It is intriguing to consider what factors influence which synapse will survive at a given endplate. For example, are the connections from any particular motor neurons at a competitive advantage over the others? The experiments we describe here address several such possibilities, pertaining to the extent of arborization, rostral/caudal spinal position, and level of activity of motor neurons.

We assessed the role of spinal position by measuring the twitch tension of motor units from different spinal roots as a function of developmental age during the period of synapse elimination in soleus muscles of normal rabbits. If the competitive ability of motor neurons were correlated with spinal position, then the relative sizes of motor units from different spinal roots should change with age. Evidence against this hypothesis has been reported for the soleus muscles of both rats and rabbits (Gordon and Van Essen, 1983; Thompson, 1983a; but see Miyata and Yoshioka, 1980). However, small differences in synapse loss could have been obscured in these studies because the data were not analyzed separately for fast and slow units; it was not recognized until later (Gordon and Van Essen, 1985) that immature fast and slow motor units differ markedly in average size, at least in the rabbit soleus. By analyzing fast and slow motor units independently and by paying particular attention to the motor units at the rostral and caudal extremes of the soleus motor pool, we have found a small, but significant difference in synapse loss according to spinal position.

If a motor neuron's arbor size influences the extent of synapse loss, there should be a systematic change in the diversity of motor unit sizes as synapse elimination proceeds. For example, if neurons with smaller arbors were at an advantage, as has been 


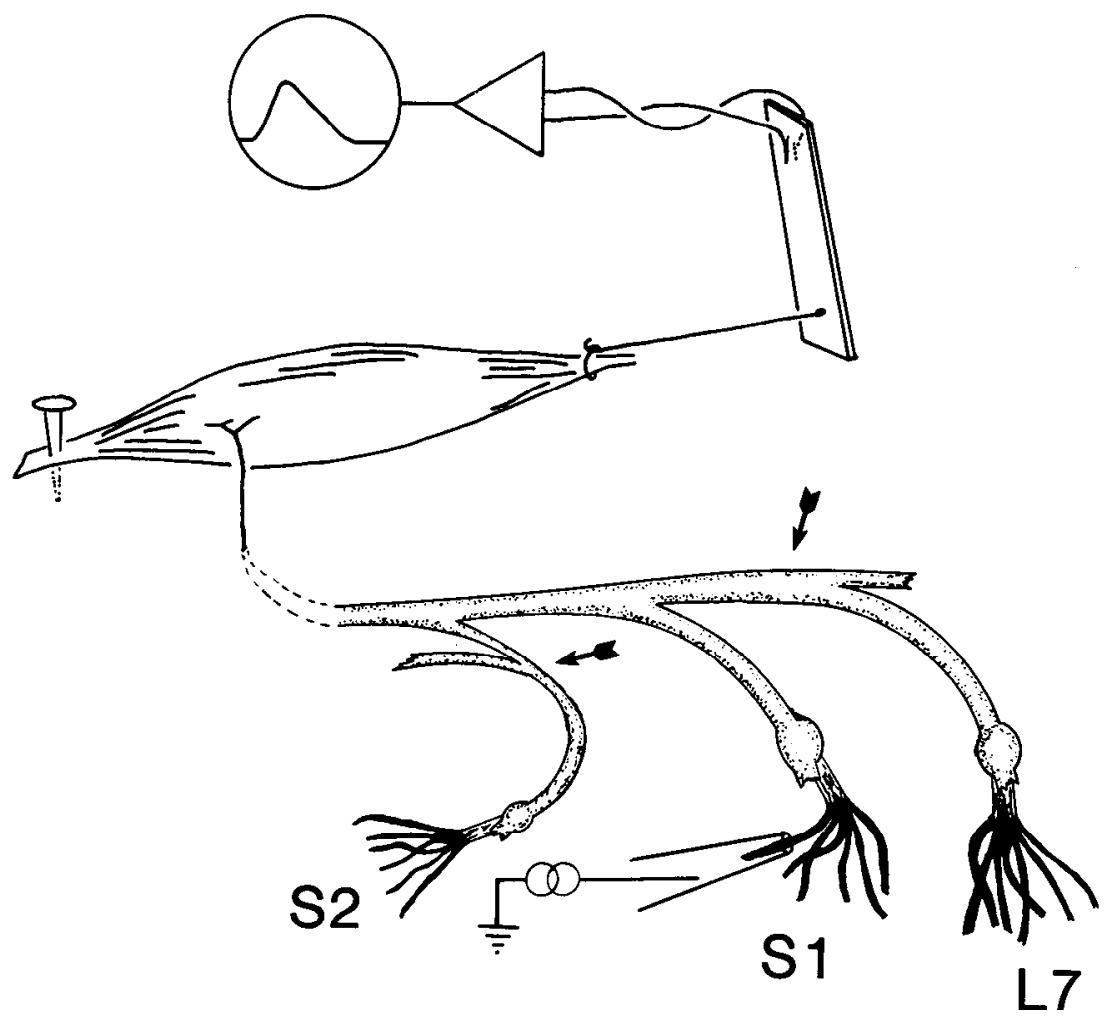

Figure 1. Diagram of the preparation for in vitro measurement of rabbit soleus muscle twitch responses. The preparation includes the rabbit soleus muscle pinned at its proximal tendon to the bottom of a wax-lined dish and its contributing innervation as far proximal as the ventral roots ( $\mathrm{L} 7, \mathrm{~S} 1$, and $\mathrm{S} 2)$; a tension transducer attached to the distal tendon via 6-0 silk thread; and a suction electrode for stimulation of teased filaments of ventral roots. $A r-$ rows indicate location in $\mathrm{L} 7$ or $\mathrm{S} 2$ spinal nerve where a TTX-laden or control plug was implanted in some animals. Modified, with permission, from Callaway et al. (1987). suggested on theoretical grounds by Smalheiser and Crain (1984), initially small motor units would lose a smaller percentage of their synapses while large units would lose proportionately more synapses. Accordingly, the diversity in motor unit sizes for the overall population would decrease. On the other hand, diversity would increase if large arbors were an advantage, a possibility suggested by the results of Gordon and Van Essen (1981). Once again, their investigations of this issue did not separate fast and slow motor unit types and therefore could not distinguish a change in diversity within a group from a systematic change in the relative size of fast and slow motor units. In the present study we found that the relative size of the 2 populations does indeed change dramatically but that there was no discernible change in the diversity within either population.

Activity has been strongly implicated in the regulation of the rate of synapse elimination. Blockage of nerve and muscle activity presynaptically, by botulinum toxin or tetrodotoxin (Thompson et al., 1979; Brown ct al., 1981), or of muscle activity alone by $\alpha$-bungarotoxin (Duxson, 1982; Callaway and Van Essen, 1989), dramatically slows or altogether halts synapse removal. Increased nerve and muscle activity, elicited by implanted stimulating electrodes, increases the rate of synapse elimination (O'Brien et al., 1978; Thompson, 1983b). However, these results do not address whether the level of activity of synapses affects their competitive ability, rather than simply governing the overall rate of the process. Will a difference in activity of synapses at the same endplate influence which synapse will remain? If the neuromuscular junction were regulated by Hebb-like rules of competition (Hebb, 1949), more active inputs should outcompete less active inputs (Ribchester and Taxt, 1983, 1984; Ridge and Betz, 1984; Ribchester, 1988b). However, we previously suggested that inactivity at least transiently increases the survival probability of a synapse when it is in direct competition with active synapses (Callaway et al., 1987). In the present study we have extended this analysis and provide evidence that the effect of inactivity substantially outlasts the period of the differential activity blockade.

\section{Materials and Methods}

Twitch tension recording. After initial sedation with inhaled methoxyflurane (Metofane, Pitman-Moore Inc.), animals were heavily anesthetized with ether, and the legs and lower spinal column were removed and placed in chilled Ringer's solution $\left(150 \mathrm{mM} \mathrm{Na}^{+}, 5 \mathrm{mM} \mathrm{K} \mathrm{K}^{+}, 1 \mathrm{mM}\right.$ $\mathrm{Mg}^{2+}, 5 \mathrm{~mm} \mathrm{Ca}^{2+}, 167 \mathrm{mM} \mathrm{Cl}^{-}, 16 \mathrm{~mm}$ glucose, $14 \mathrm{~mm}$ HEPES buffer). The soleus muscle was dissected free along with its contributing innervation up to and including the ventral roots L7, S1, and S2 (Fig. 1).

Twitch tensions resulting from stimulation of each of the whole ventral roots contributing to the soleus muscle were determined. Roots were then split into 10-30 filaments, and twitch tensions and rise times of individual motor units were determined by graded stimulation of individual filaments of ventral roots containing 4 or fewer soleus motor axons (Fig. 1). Twitch responses measured with a tension transducer were amplified, converted to digital signals, and analyzed both on-line and off-line on an IBM PC-XT computer. When a filament contained more than one soleus motor axon, the summed responses were subtracted to reveal the twitch responses of the individual motor units. To assess the unitary nature of each motor unit response, we routinely adjusted the stimulus strength to be at the unit's threshold and observed that the measured tension fluctuated between 2 distinct levels several times, without any change in the stimulus. We also measured the twitch tensions elicited by direct, supramaximal stimulation of the whole musclc. The cxpcriment was discontinucd if the maximal response dropped below $80 \%$ of its original value, and maximal responses were measured frequently enough that the value seldom varied by more than $5 \%$ between samples.

In general, every motor unit was measured in ventral roots containing fewer than 20 soleus motor axons, but it was usually necessary to terminate the experiment before all motor units from the $\mathrm{S} 1$ ventral root could be assayed. Typically, a total of 30-50 motor units were assayed per muscle. Measurements from the various ventral roots were interspersed in order to avoid artifactual size differences of motor units that might result from systematic drifts in recording conditions or state of 
the preparation over time. Complete interspersal was difficult to achieve, though, especially for roots having only a few motor units. To address this issue further we occasionally assayed an identified motor unit again at the conclusion of an experiment (1-2.5 hr later). The average size for the second measurement was $95 \%$ of the first, indicating no consistent decline. In 7 of 9 instances of this type, the change in measured size was less than $12 \%$, which was not markedly greater than the fluctuations encountered during repeated measurements made in succession. Also, when the mean size of the first 5 fast or slow motor units measured from the S1 root of each animal was compared with the mean of the last 5 , there was no systematic tendency for measured tensions to increase or decrease. Overall, the last 5 units measured were smaller than the first by only $4 \%$ for fast and $12 \%$ for slow; neither difference is statistically significant ( $p>0.2$ for each comparison). Even if these small differences were meaningful, a modest decline in mean sizes over the course of an experiment would work against the observed differences in size between motor units from different roots (see Results). For example, we found that in normal animals the motor units from the minor contributing roots were relatively small, even though they tended to be assayed early during the experiments. For TTX-implanted animals, the motor units from the implanted roots were measured relatively late in the sequence (owing to the need to wait for TTX washout at the intermediate age and explicitly to avoid the possibility of bias at the late age); nonetheless, their mean sizes were relatively large. We noted only modest changes in twitch rise times when repeated measures were made from identified motor units or more frequently from the whole muscle. Small changes in rise time should not influence twitch type assignment since a secondary criterion was available in the rare cases when twitch type was ambiguous based on rise time alone (see below). Additional aspects of tension recordings are described elsewhere (Gordon and Van Essen, 1983, 1985; Soha et al., 1989).

Separation of motor unit twitch types. The distinction between fast and slow motor units was based mainly on the bimodal distribution of twitch rise times characteristic of rabbit soleus muscles at all postnatal ages (Fig. 2; see also Gordon and Van Essen, 1985). In some muscles the best split point for designating fast versus slow motor units was not obvious (even though the distribution of rise times was bimodal); in such cases, there was uncertainty about the assignment of several $(\leq 5)$ motor units. To assign these borderline motor units more accurately, we took advantage of the approximately 2 -fold difference in mean motor unit twitch tension between fast and slow groups (Gordon and Van Essen, 1985; see Results); the initially ambiguous units were assigned to the group whose range of sizes best matched the unit in question.

Grouping of animals. Rabbits were categorized according to their age at the time of in vitro physiology (early, intermediate, or late), experimental treatment (normal, control-implanted, or TTX-implanted), and the distribution of soleus motor axons among the ventral roots (Table 1).

The ages examined were 4-5 d postnatal for the early group and 8$9 \mathrm{~d}$ for the intermediate group. The late group included $11-15 \mathrm{~d}$ normal animals but only 14 and $15 \mathrm{~d}$ animals for control and TTX-implanted groups.

In all muscles, the innervation to the soleus arose from 2 or 3 spinal roots. The $\mathrm{S} 1$ root invariably provided the majority of the inputs, as judged by comparing the overall tension elicited from stimulation of different roots and/or by counting the number of motor units isolated from each root. In about half of the cases (17 of 33 normal muscles), the additional inputs arose from both $\mathrm{L} 7$ and $\mathrm{S} 2$ roots; in the other cases, the additional inputs usually arose from $\mathrm{L} 7$ alone ( 12 cases) but sometimes were from S2 alone (4 cases). For simplicity, we will refer to $\mathrm{L} 7$ and $\mathrm{S} 2$ as "extreme roots" and $\mathrm{S} 1$ as the "middle root," even for muscles supplied by only 2 roots.

In the experiments aimed at studying the effects of differential activity, our experimental paradigm required that active and inactive synapses compete directly against one another on active muscle fibers. For this condition to be met, the contribution from the implanted nerve had to be small, so that at the time of the initial TTX implant each muscle fiber with an inactive input would also receive one or more active inputs. We therefore analyzed the innervation of normal 4-5 d rabbits to determine how many soleus motor axons an extreme root could contain while still having all of its target fibers co-innervated by the remaining roots. We found that the overlap in innervation at this early age was complete as long as the extreme root contained 18 or fewer soleus motor axons (see Results). This was the case in the great majority of animals examined; extreme roots meeting this condition were termed "minor roots." In about $20 \%$ of the animals (15/89), one of the extreme roots

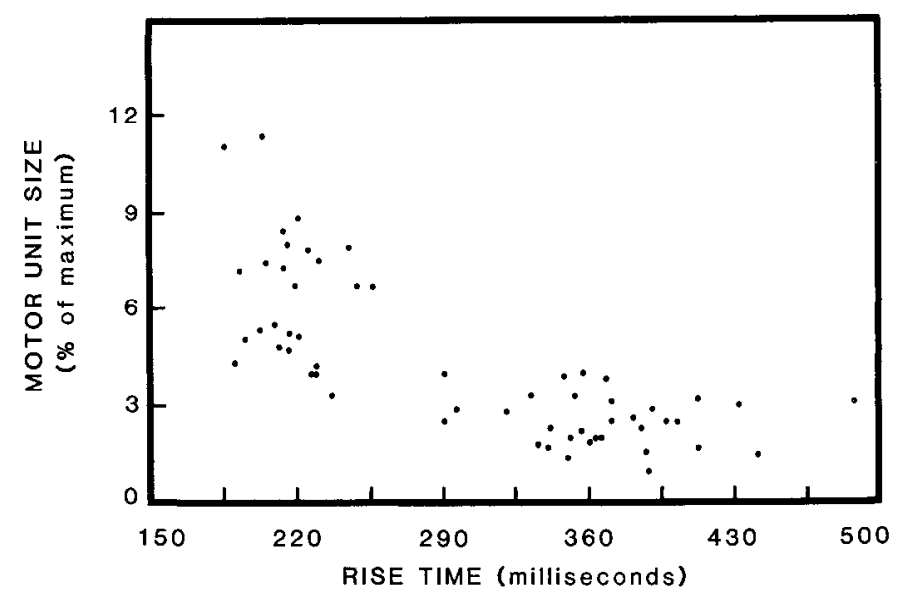

Figure 2. Plot of twitch rise times against sizes of motor units, expressed as percentage tension, from a normal 8 -d-old animal. Mean percentage tension motor unit sizes were $6.4 \pm 0.4$ and $2.5 \pm 0.1 \%$ for fast and slow units, respectively. Mean rise times were $218 \pm 3$ and $368 \pm 8 \mathrm{msec}$ for fast and slow units, respectively. The fastest rise time for a slow unit was $291 \mathrm{msec}$, and the slowest rise time for a fast unit was $257 \mathrm{msec}$. The rise times for these motor units are much slower than in vivo owing to the lower temperature at which our in vitro measurements are made.

was estimated to contain more than 18 soleus motor axons and was termed a "major root." The 3 cases involving a successful block of a major root were analyzed separately from the rest of the data. In normal and control animals, cases with soleus innervation from major rools were discarded without further analysis because only the minor root cases formed an appropriate basis for comparison with the main group of TTX-implanted animals.

Analysis of twitch tension data. We were mainly interested in comparing the average size of motor units originating from different spinal roots (e.g., extreme versus middle roots; active versus inactive roots). We based our analysis on 2 different expressions for motor unit twitch tension. The first is the "percentage tension," which involves scaling to the maximal tension elicited by direct muscle stimulation:

$$
t_{i}=100\left(\theta_{i} / \Theta_{\max }\right)
$$

where $\theta_{i}$ is the twitch tension in grams of the $i$ th motor unit, $\Theta_{\max }$ is the maximal direct tension in grams, and $t_{i}$ is the percentage tension.

Scaling to a percentage value was intended to compensate for the large variability in maximal tension produced by different muscles, even for muscles from animals of similar age. However, even after this initial compensation, large ( 2 -fold or more), differences persist in the average percentage tension of both fast and slow motor units from different muscles (see Results). Hence, for much of our analysis we used an additional normalization step in which the motor unit sizes were scaled to the median value for the corresponding contractile type from the middle root of the same animal:

$$
\phi_{i}=100\left(t_{i} / T_{m m}^{\mathrm{s}, \mathrm{f}}\right)
$$

where $\phi_{i}$ is the "normalized tension" and $T_{m m}^{\mathrm{s}}$ and $T^{\mathrm{f}}{ }_{m m}$ are the median percentage tension values for the fast and slow motor units from the middle root of the same animal. In this and other equations, variables represented by lowercase symbols indicate that they represent a size estimate based on the twitch response of a single motor unit, while representation by uppercase symbols indicates values calculated from a population of motor units. Scaling was done to medians rather than means because subsequent statistical analyses were carried out using nonparametric tests. In order to assure that the median values estimated for each animal gave an adequate estimation of the true median value, only animals from which at least 5 middle root motor units of each twitch type were measured were included in our analysis.

The normalized tension emphasizes a motor unit's size relative to the typical middle root motor unit in the animal from which it was measurcd, and it provides a satisfactory basis for pooling results from different animals. For statistical analysis of normalized tension values, all the appropriate values from all the animals in a group were pooled 
Table 1. Number of animals by experimental group, age, and contributing ventral roots

\begin{tabular}{|l|ccc|cc|c|}
\hline & \multicolumn{3}{|c|}{ Normal } & \multicolumn{2}{c|}{ Control } & TTX-implanted \\
& L7 & S2 $\quad$ L7\&S2 & L7 implant S2 implant & L7 implant \\
\hline Early (4-5 days) & 2 & 0 & 5 & -- & 4 \\
\hline $\begin{array}{l}\text { Intermediate } \\
\text { (8-9 days) }\end{array}$ & 0 & 3 & 3 & 6 & 4 & $4(3)$ \\
\hline $\begin{array}{l}\text { Late } \\
\text { (11- or 14-15 days) }\end{array}$ & 4 & 1 & 4 & 9 & 0 & 11 \\
\hline
\end{tabular}

Number of animals by experimental group-normal, control, and TTX-implanted at eariy, intermediate, and late ages-and by ventral roots contributing to the soleus innervation. For normal animals, the first number in each group represents the number of animals in that group whose soleus innervation was contributed by the S1 and L7 ventral roots; the second number corresponds to the number of animals with soleus contributions from S1 and S2; and the third number is the number of animals with soleus contributions from all 3 ventral roots. For TTX-and control-implanted animais the first number for each group corresponds to the number of animals whose $\mathrm{L} 7$ spinal nerve was implanted and the second, the number of animals with an $\$ 2$ implant. Only animals for which there was a soleus contribution from the implanted nerve are included. Some animals were not analyzed and are not included in the above table because of one of the following: fewer than 5 middle motor units of each twitch type were assayed before the in vitro preparation degraded, equipment malfunction made motor unit size estimates inaccurate, or the extreme root had too many motor units to be considered a minor root, so motor unit sizes were not assayed. Three animals for which the extreme root was not a minor root are included in parentheses for S2 TTX-implanted animals at the intermediate age; these animals were analyzed and are considered separately as TTX-implanted, major block animals in the results section

together, and comparisons of those populations were made with the Mann-Whitney $U$ test or the Kolmogorov-Smirnov test. The overall mean normalized tension value for each group was thus calculated by

$$
\Phi=\left(\sum_{m} \sum_{i} \phi_{m i}\right) / n_{\mathrm{tot}},
$$

where $\phi_{m i}$ is the value of $\phi$ for the $i$ th unit from the $m$ th muscle, $n_{\text {tor }}$ is the total number of units, and $\Phi$ is the mean normalized tension for the experimental group.

When it was necessary to compare absolute measures of motor unit size without normalization to the median (i.e., using percentage tension values), it was not appropriate to pool the values from different animals and treat them as independent samples-the large variation in mean sizes between animals made each value dependent on the animal from which it was measured. We therefore calculated the mean percentage tension values from individual animals:

$$
T_{m}=\left(\sum_{i} t_{i}\right) / n,
$$

where $n$ is the number of units and $T_{m}$ is the mean percentage tension for the $m$ th muscle. These mean percentage tension values were pooled for the various experimental groups, and their distributions were compared using the Mann-Whitney $U$ test. The overall mean percentage tension value for each group was calculated by

$$
T_{\text {tot }}-\left(\sum_{m} T_{m}\right) / n,
$$

where $n$ is the number of muscles in the experimental group and $T_{\text {tot }}$ is the overall mean percentage tension.

TTX and control plug manufacture. TTX-laden plugs were manufactured by the method developed by Gordon (1984) and described by Callaway et al. (1987). Silastic plugs containing $0.64-0.73 \mu \mathrm{g}$ TTX were formed on the tip of a 2-3 mm length of 40 gauge platinum wire and cured for $3 \mathrm{~d}$ at room temperature and at least 4 additional days at $5^{\circ} \mathrm{C}$. Potency was consistent among plugs made at the same time and under the same conditions. However, despite careful measurement of the amount of TTX applied, the potency of different batches of plugs was quite variable. With overly potent plugs, the animals failed to recover from anesthesia; with underpotent plugs there was no muscle paresis in the behavioral assay described below. If a batch of plugs proved too potent when first implanted, the remaining plugs were stored at $5^{\circ} \mathrm{C}$ for several weeks and implanted when the Silastic had cured further and perhaps some of the TTX had degraded. Control plugs were made identically except no 'I"IX was applied.

Plug implantation. A control or TTX-laden plug was surgically implanted into the left $\mathrm{L} 7$ or $\mathrm{S} 2$ spinal nerve of 4 - or 5 -d-old rabbits under ketamine anesthesia (120 mg/kg, i.m.). For S2 implantation, the spinal nerve was exposed by incision through the skin and musculature alongside the dorsal spinal cord just above the nerve's exit from the vertebra, and a plug was implanted just proximal to the junction at the sciatic plexus. L7 implantations were achieved by a ventral approach just lateral to the lower abdominal cavity; a plug was implanted into the $\mathrm{L7}$ spinal nerve distal to its juncture with the L6 spinal nerve and proximal to its juncture with S1. Plugs were inserted into the appropriate nerves through a small hole in the epineurium made with a fine tungsten needle. After allowing at least $2 \mathrm{hr}$ for recovery, rabbits were returned to a hutch with their littermates to be cared for by their mothers.

Assays for $T T X$-induced activity block. The effectiveness of nerve impulse blockade in TTX-implanted nerves was assessed both in vitro and in vivo. The in vitro assay was the more direct, as it tested the ability of the affected nerve to propagate action potentials past the implant site. All TTX- and control-implanted animals were assayed for conduction block of their implanted nerve in vitro during and/or after dissection of the soleus muscle and its innervation. The implanted nerve was stimulated both proximal and distal to the implant with a roving bipolar stimulating electrode $(5-15 \mathrm{~V}, 0.1-0.2 \mathrm{msec})$. The nerve was considered blocked if the distal stimulation induced visible muscle contraction (not necessarily limited to the soleus muscle) but proximal stimulation did not. For animals that proved blocked based on this assay, the TTX plug was removed, and motor unit twitch responses were measured after conduction was restored (20-120 min later).

Since our in vitro assessments were made at about $14-19^{\circ} \mathrm{C}$, they do not necessarily indicate that conduction was fully blocked at higher temperatures in vivo. We therefore used an in vivo assay as well. Animals were inspected for left (implanted) versus right side asymmetry in foot position at rest and in resistance to extension. In vivo assays of S2implanted animals proved unreliable because of the small number of motor axons in that nerve and its primary innervation of tail musculature. However, in vivo assays of L 7-implanted animals proved very reliable. L7-blocked animals were characterized by a moderate to strong resting extension of the left foot relative to the right when the animal was held on its back, and by little or no reflexive counterflexion in 


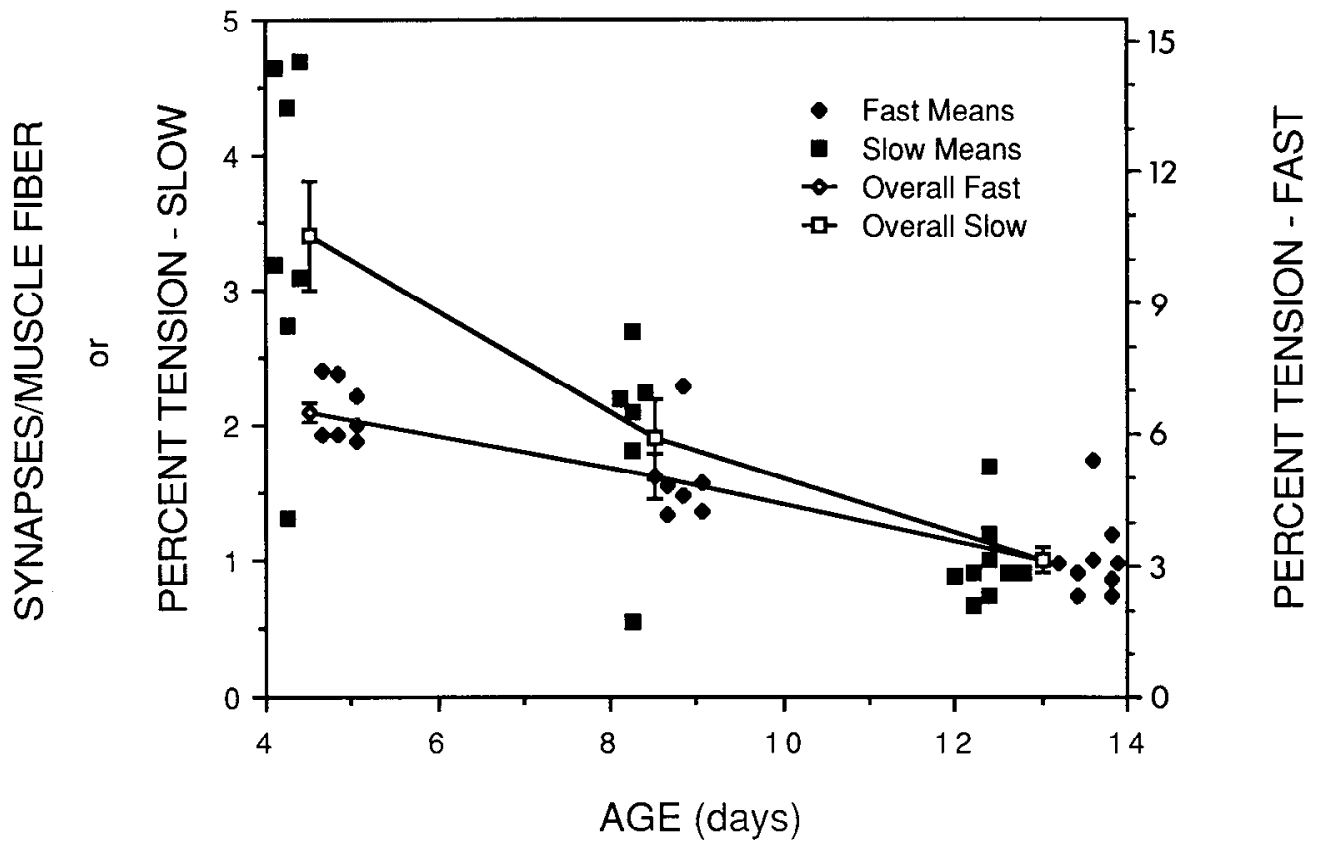

Figure 3. Mean motor unit sizes, expressed as percentage tension, from middle roots of individual early, intermediate, and late age animals, along with the average degree of polyinnervation expressed as synapses per muscle fiber. Filled diamonds and squares, respectively, represent mean values for fast and slow motor units from individual animals. Open symbols represent overall mean values. The same scale for synapses per muscle fiber is used for both fast and slow values, but the percentage tension values are scaled differently for the 2 populations so that both overall mean values correspond to unity on the polyinnervation scale at the late age. Note that for the slow population the percentage tension scale is the same as the synapses per muscle fiber since the overall mean slow percentage tension at the late age was 1.0 . Mean percentage tension was greater for fast than for slow motor units for every animal at every age, although in a few cases the mean slow value from one animal was larger than the mean fast size from a different animal of the same age. There is extensive variability in mean percentage tension, even for a single twitch type, between different animals of the same age. Possible sources of biological variability include individual differences in the total numbers of motor axons or muscle fibers, in the relative numbers of fast and slow motor neurons and of fast and slow fibers, and in the average degree of polyinnervation. The most likely source of measurement error stems from underrepresentation of maximal tension due to nonisometric recording conditions (Brown and Matthews, 1960). This would result in an overestimate of the percentage of soleus muscle fibers innervated by individual motor neurons as estimated by the percentage tension. We estimated the degree of this error and its variability by multiplying the average percentage tension from middle roots of late age muscles by the number of soleus motor neurons, estimated to be about 65 by Bixby et al. (1980). This value averaged 131 $\pm 33 \%$ (mean $\pm \mathrm{SD}$ ) for the 18 late-aged normal and control muscles, whereas it should be precisely $100 \%$ if there were no residual polyinnervation and if there were perfect isometric recording conditions.

response to passive extension of the foot. The reliability of this assay is evidenced by comparison of in vivo and in vitro assessments of the state of blockade made in succession on the same day. Fourteen animals were judged to be blocked by the in vivo assay $4 \mathrm{~d}$ after the $\mathrm{L} 7$ nerve (or sometimes S1 or L6 inadvertently) had been implanted with a TTX plug. In each of these cases the implanted nerve was also blocked based on the in vitro assay. An additional 2 animals were blocked in vitro but not in vivo on the fourth day: one, a block of L7, had been clearly blocked in vivo for $3 \mathrm{~d}$; the other was a block of $\mathrm{S} 1$ that showed only weak evidence of in vivo block for 1-2 d. Dozens of other TTX-implanted animals were blocked based on the in vivo assay for 1-3 d but were not blocked based on either the in vivo or the in vitro assay on the fourth day. TTX-implanted animals that were not blocked based on the in vitro assay at the time of dissection were discarded without further analysis.

No control-implanted animals showed evidence of conduction block in the implanted nerve based on either of the assays. No animal showed evidence of TTX block beyond $5 \mathrm{~d}$ after implantation. Therefore, for the long-term TTX series, only L7 was implanted, animals were dissected at days 14-15 (10-11 d after implantation), and only animals blocked for 4-5 d by the in vivo assay were dissected and analyzed.

Silver staining of nerve terminals. Muscles in the late age control and TTX-implanted groups were stained by a modification of the silvercholinesterase method described by Hopkins et al. (1985). Following fixation and storage in buffer solution as they describe, muscles were cut longitudinally into $40 \mu \mathrm{m}$ sections on a freezing microtome. Sections were mounted on gelatin-subbed slides. Mounted sections were placed in cold Karnovsky AChE stain (Karnovsky and Roots, 1964) with no substrate for 6-7 min and then washed in water for $5 \mathrm{~min}$. Sections were then stained for esterase to mark endplates for $15-20$ min at $37^{\circ} \mathrm{C}$ as described by Pestronk and Drachman (1978). This stain produces a transparent blue reaction product that allows visualization of nerve terminals within the esterase-stained region. Sections were subsequently processed as described by Hopkins et al. (1985).

One hundred endplates from each muscle were observed at $400 \times$ magnification, and the number of axons contributing to the innervation of each endplate was determined. Slides were coded so that the observer did not know whether a given muscle was from the TTX-or controlimplanted group. Endplate-rich regions of muscle sections were examined at $400 \times$. Every endplate, as defined by esterase staining in the resulting field, was examined, but only complete endplates not at the edges of the section plane (determined by focus) were scored. When all endplates in the field had been observed, the stage was moved to bring more endplates into view and every additional endplate was characterized. Typically 30-60 endplates were observed per section.

\section{Results}

Part I. Normal animals: synapse elimination by fiber type and spinal position

\section{Changes in polyinnervation by fiber type}

We begin by analyzing overall changes in fast and slow motor units among normal animals without regard to spinal position. Figure 3 shows, for individual normal animals, the mean percentage tension of fast (filled diamonds) and slow motor units (filled squares) from $\mathrm{S} 1$, the middle root $\left(\mathrm{T}_{m}^{\mathrm{f}}\right.$ and $\mathrm{T}_{m}^{\mathrm{s}}$, Equation 
Table 2. Mean motor unit sizes expressed as normalized tension and as percentage tension by experimental group, age, contributing ventral root, and twitch type

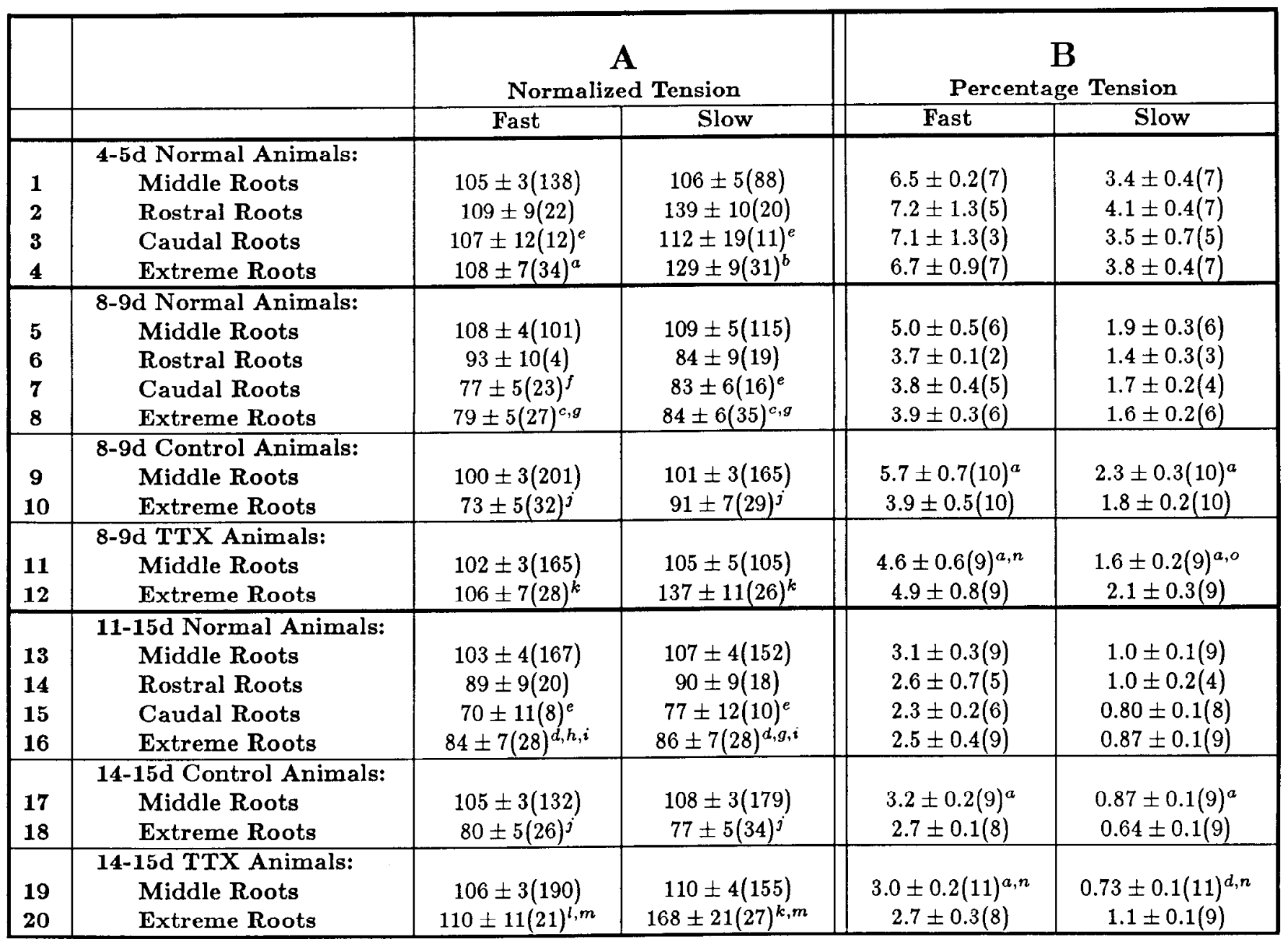

Column A. Normalized values represent means \pm SEM of the pooled motor unit sizes, expressed as a percentage of the median value for the middle root, from all animals in the group (see Materials and Methods, Equation 3). Numbers in parentheses are the numbers of motor units. The normalized values from middle roots exceed $100 \%$ because distributions of motor unit sizes are skewed such that the mean is greater than the median (see Figs. 5-7). Column B. Percentage tension values represent means \pm SEM of the mean values from each animal in the group (values shown in Fig. 3; see Materials and Methods, Equation 5). Numbers in parentheses represent the numbers of animals (means) in each group. The number of animals for rostral, caudal, and extreme root groups are sometimes smaller than for middle root groups because not every animal has soleus contribution from both rostral and caudal roots and some extreme roots contributed unit(s) of one twitch type but not the other.

"Equal to same age, normal, middle root; $p>0.2$

"Larger than same age, normal, middle root; $p<0.05$.

"Smaller than same age, normal, middle root; $p<0.001$.

"Smaller than same age, normal, middle root; $p<0.05$.

Equal to the same age, normal, rostral root; $p>0.2$.

Fqual to the same, age, normal, rostral root; $p>0.1$.

" Smaller than early age, normal, extreme root; $p<0.001$.

"Smaller than early age, normal, extreme root; $p<0.005$.

'Equal to intermediate age, normal, extreme root; $p>0.2$.

Equal to same age, normal, extreme root; $p>0.2$.

${ }^{*}$ Larger than same age, control-implanted, extreme root; $p<0.001$.

' Larger than same age, control-implanted, extreme root; $p<0.02$.

${ }^{m}$ Equal to intermediate age, TTX-implanted, extreme root; $p>0.2$

${ }^{n}$ Equal to same age, control-implanted, middle root; $p>0.2$.

"Smaller than same age, control-implanted, middle root; $p<0.05$.

All $p$ values determined from Mann-Whitney $U$ test.

4). Also shown are the overall means ( \pm SEM) for each age group ( $T_{\mathrm{tot}}^{\mathrm{r}}$ and $T_{\text {tot }}^{\mathrm{s}}$, Equation 5; open diamonds and open squares for fast and slow, respectively). The fast and slow means are plotted on different scales, which were adjusted to make the overall means coincide at the late age. At the early age, the overall mean percentage tension values were about twice as large for fast units as for slow units (6.5 vs $3.4 \%$ ). These values decreased to 3.1 versus $1.0 \%$ for fast and slow motor units at the late age (see 

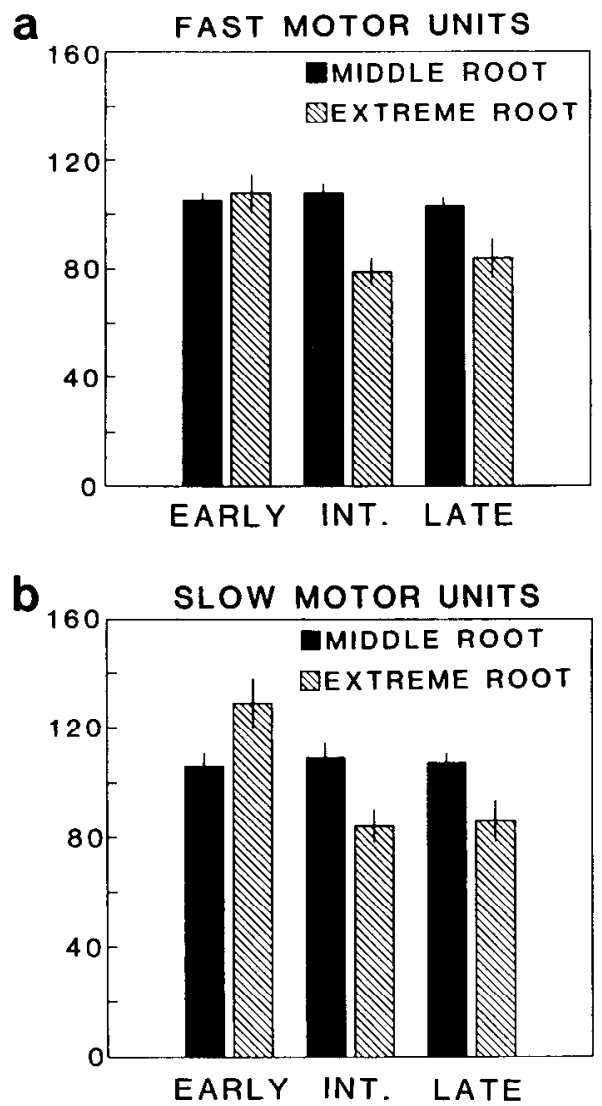

\section{MOTOR UNIT TWITCH STRENGTH AS \% OF MIDDLE ROOT MEDIAN MEAN PLUS/MINUS S.E.M.}

Figure 4. Comparisons of normalized fast motor unit sizes $(a)$ and slow motor unit sizes $(b)$ from middle and extreme roots of early-, intermediate-, and late-age animals. Bar heights represent means \pm SEM normalized motor unit sizes (percentage of middle root median). At the early age, for both fast and slow groups, extreme root motor units were not smaller than middle root motor units. However, 4-5 d later, at the intermediate age, extreme root motor units were significantly smaller than middle root motor units for each twitch type. This difference persisted in singly innervated late-age animals. When scparate statistical comparisons of normalized mean sizes for rostral and caudal motor units against middle motor units were made, the only significant difference ( $p<0.05$, Mann-Whitney $U$ test) at the early age was for the slow, rostral units $(139 \pm 10 \%$, Table $2,2 \mathrm{~A})$, which were larger on average than the slow middle units (106 $\pm 5 \%$, Table 2, 1A). At intermediate and late ages, each of the 4 differences (fast and slow at each age) for caudal versus middle motor units was statistically significant with caudal means smaller than middle. However, for rostral versus middle motor units there was a statistically significant difference for only 1 of the 4 comparisons-slow motor units at the intermediate age. The differences between rostral and middle motor units were statistically significant for both the fast and slow populations if the values from the intermediate- and late-age groups were pooled. This was also the case for both intermediate and late ages if fast and slow values were pooled.

Table 2, column B). Similar results were obtained by Gordon (1984). The difference between fast and slow motor unit tensions could result from a greater number of muscle fibers innervated by each neuron for the fast population or from generation of more tension per muscle fiber for the fast fibers.

Since nearly all muscle fibers are singly innervated at the late age (Bixby and Van Essen, 1979), the ratio of percentage tensions between early and late ages provides an estimate of the average degree of polyinnervation of each fiber type. This ratio is indicated by the scale on the left of Figure 3. Interestingly, the estimated rate of synapse loss between early and late ages is about 2 -fold greater for the slow than the fast fibers $(0.28 \mathrm{vs}$ 0.13 synapses/fiber/d). Also the rate of elimination appears to be similar during the different time periods we tested for the fast fibers $(0.12 /$ fiber/d between early and intermediate ages vs 0.14 between intermediate and late) but much faster during the earlier than during the later interval for the slow fibers $(0.38 /$ fiber/d vs 0.20 ). It is important to note, however, that these estimates of the rate of synapse elimination are predicated on the unproven assumption that the relative tension generated by individual fast and slow muscle fibers does not change during this period (see Gordon, 1984).

It is apparent from Figure 3 that there was considerable variability from one muscle to the next in the average percentage tension of motor units from each contractile type and cach agc group. The ratios of the largest and smallest average percentage tension for a given contractile type and age group ranged from nearly 2 -fold to more than 5 -fold. Some of this variability must arise from sampling errors, given that we assayed only a fraction of the whole motor unit pool and that there was considerable dispersion of motor unit tensions within each muscle (cf. Fig. 2 and below). An analysis of variance, however, indicated that the differences between muscles were statistically significant and were not entirely attributable to incomplete sampling. Possible sources of between-animal variation are noted in the legend to Figure 3.

The variability within animals and that between animals comprise 2 distinct, separable sources of statistical fluctuation. Our primary means of achieving this separation was to pool the data on motor unit tensions only after scaling to the median value for the middlc root from the same animal (see Materials and Methods, Equations 2 and 3). This normalization procedure largely removes the contribution of individual variability, whether it be from measurement errors or of biological origin. It thereby allows for sensitive comparisons, since motor units from different muscles (but from same root and contractile type) can be treated as independent samples from a single distribution. We also used the alternative method of calculating the mean percentage tension values for each root and contractile type from each muscle (see Materials and Methods, Equation 4). When a mean percentage tension value for a group of motor units from different muscles was desired, it was calculated by pooling the means from the individual muscles (Equation 5). While percentage tension is a more direct measure than normalized tension, it is inherently less sensitive to small (but genuine) differences in the relative sizes of motor units from the same animal.

\section{Changes in extreme relative to middle motor unit sizes}

In comparing motor units from different spinal roots, we found that the normalized motor unit tensions from extreme roots (L7 and S2) were on average significantly smaller than those from the middle root (S1) at both intermediate and late ages. This difference was not present in the early age group. These findings are illustrated in Figure 4, which shows bar histograms of mean normalized motor unit tensions. In addition, Table 2 shows these and other data expressed as normalized tension (column A) and as percentage tension (column B).

For fast motor units (Fig. $4 a$ ), the normalized tensions from extreme roots (hatched bars) and middle roots (solid bars) were not significantly different on average at early ages (108 vs $105 \%$, 

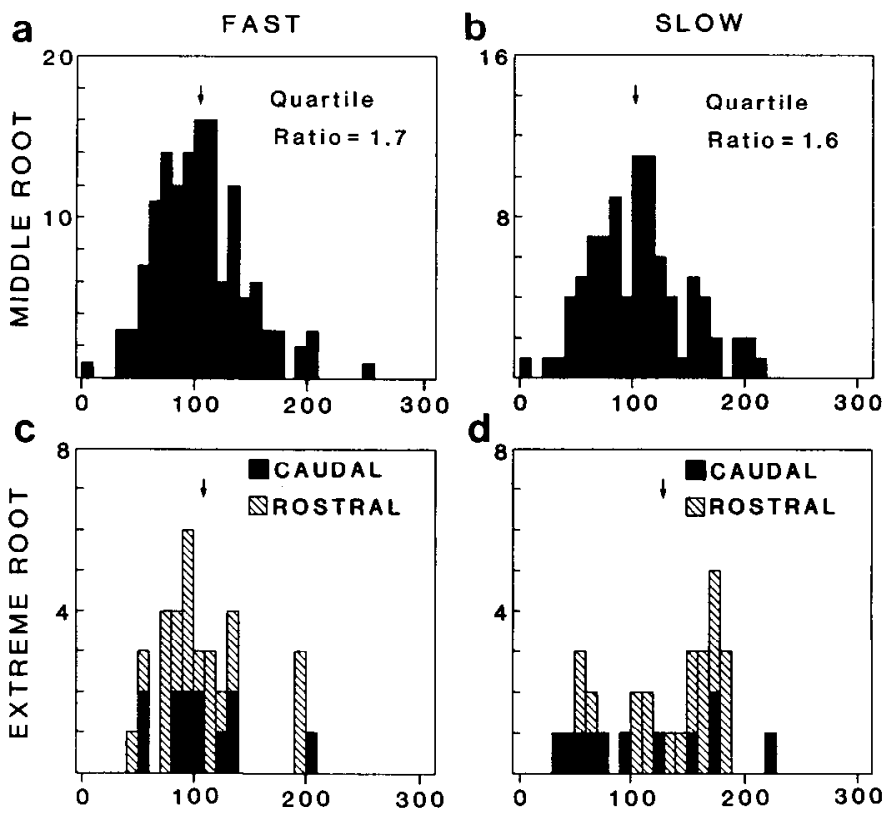

MOTOR UNIT TWITCH STRENGTH

\section{AS PERCENT OF MIDDLE ROOT MEDIAN}

Figure 5. Distributions of normalized motor unit sizes for fast $(a, c)$ and slow $(b, d)$ groups from middle $(a, b)$ and both rostral and caudal extreme $(c, d)$ roots of early age animals. Arrows indicate means. Note the similarity in size of motor units from rostral versus caudal and extreme versus middle roots, with the exception of slow, extreme motor units being significantly larger than slow middle root motor units.
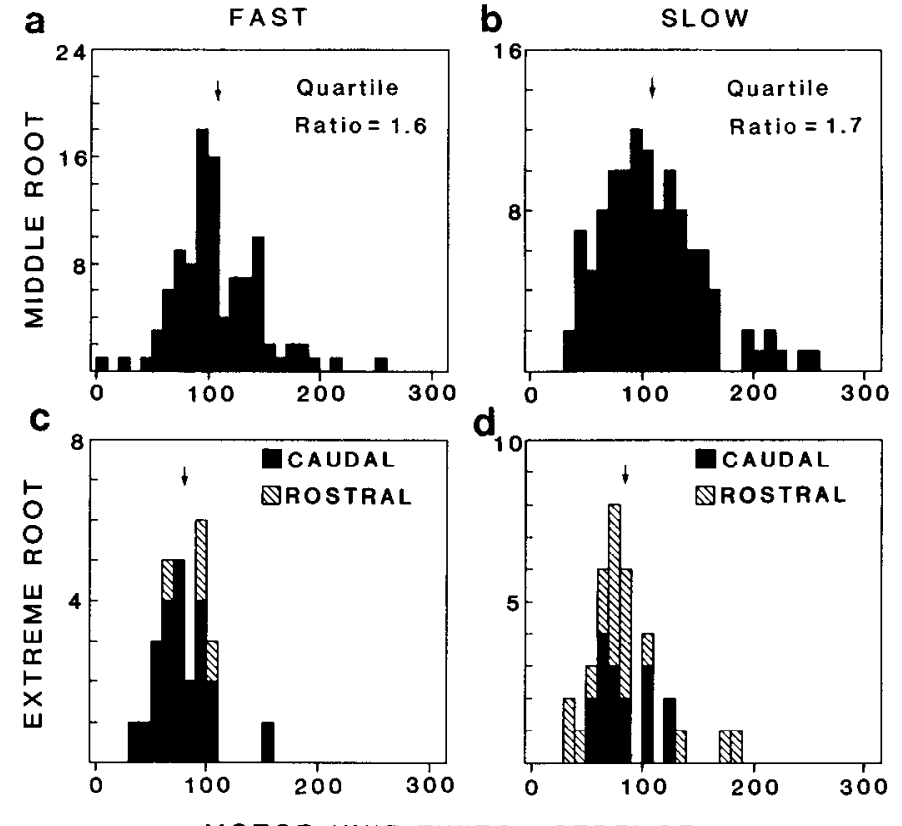

MOTOR UNIT TWITCH STRENGTH

AS PERCENT OF MIDDLE ROOT MEDIAN

Figure 6. Distributions of normalized motor unit sizes for fast $(a, c)$ and slow $(b, d)$ groups from middle $(a, b)$ and both rostral and caudal extreme $(c, d)$ roots of intermediate age animals. Arrows indicate means. Note the similarity in size of motor units from rostral versus caudal roots and the smaller size of motor units from extreme roots versus middle root motor units. $p>0.2$, Mann-Whitney $U$ test). At the intermediate age, however, fast motor units from extreme roots were on average $27 \%$ smaller than those from middle roots (79 vs 108\%, $p<0.001$ ). The difference was not as large for 11 - to 15 -d-old animals $(84 \%$ for extreme vs $103 \%$ for middle roots), but it was still statistically significant $(p<0.05)$.

The results for the slow population (Fig. $4 b$ ) were similar to those from the fast population, except that at early ages extreme root motor units were actually larger than those from middle roots by a significant amount ( 129 vs $106 \%, p<0.05$ ). Again comparing hatched to nonhatched bars from the same age, slow motor units from extreme roots were on average $23 \%$ smaller than those from middle roots at intermediate ages ( 84 vs $109 \%$, $p<0.001)$ and $20 \%$ smaller at late ages $(86 \%$ for extreme vs $107 \%$ for middle, $p<0.05$ ).

By comparing the hatched bars at different ages, it is apparent that the relative size of extreme motor units declines between early and intermediate ages and then remains stable between the intermediate and late ages. This initial decline is somewhat larger for the slow population (from 129 to $84 \%$ ) than for the fast population (from 108 to $79 \%$ ).

These results suggest that synapse elimination initially proceeds faster for motor neurons from extreme roots compared with those from the middle root. But how much of a difference is this in relation to the overall rate of synapse elimination in either root? To address this issue we used percentage tension values (Table 2, column B) to estimate the overall decline in motor unit sizes for middle and extreme roots. For the fast population, the decline in motor unit size between early and intermediate ages was only $23 \%$ for the middle root (Table 2, $\mathrm{AB}$ vs $5 \mathrm{~B}$ ), whereas the decline was nearly twice as great (42\%) for the extreme roots (Table 2, 4B vs $8 \mathrm{~B}$ ). For the slow population during the same period, the decline in motor unit size was even greater for the extreme roots $(58 \%$; Table 2 , 4B vs $8 \mathrm{~B})$, but this was only modestly (1.3 times) greater than the decline for middle roots (44\%; Table $2,1 \mathrm{~B}$ vs $5 \mathrm{~B}$ ). In other words, for the slow motor neurons the estimated rate of synapse loss is high overall, with only a moderate fractional difference between extreme and middle roots, whereas the rate is considerably lower for the fast population, but with a greater difference between extreme and middle roots.

\section{Rostral versus caudal motor unit sizes}

Figures 5-7 show histograms of the distributions of normalized motor unit tensions for middle roots (upper panels) versus extreme roots (lower panels) for fast and slow populations (left and right panels, respectively) for each age group. In the lower panels, bars are shaded differently for motor units from rostral (hatching) and caudal (solid) roots. Although there appears to be a trend for motor units from rostral roots to be larger than those from caudal roots ( $13 \%$ on average), the motor units were indistinguishable in size $(p>0.1)$ for both fast and slow populations from all age groups, whether analyzed separately or combined (see also Table 2). 
LATE AGE ANIMALS
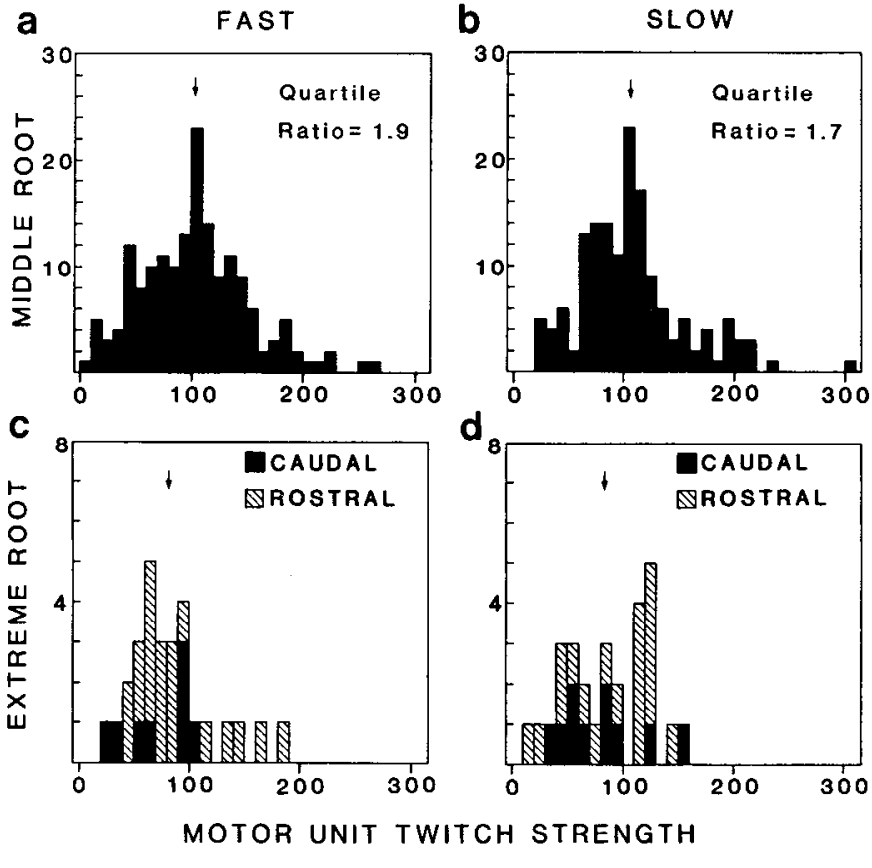

AS PERCENT OF MIDDLE ROOT MEDIAN

Figure 7. Distributions of normalized motor unit sizes for fast $(a, c)$ and slow $(b, d)$ groups from middle $(a, b)$ and both rostral and caudal extreme $(c, d)$ roots of late-age animals. Arrows indicate means. As at the intermediate age, motor units from rostral versus caudal roots have a similar distribution, whereas motor units from extreme roots are skewed towards smaller values than middle root motor units.

\section{Distributions of middle motor unit sizes from normal animals}

If a motor neuron's arbor size influences its ability to compete for retention of synapses, the diversity in motor unit sizes should change as synapse elimination proceeds. Our basic measure of diversity was the quartile ratio (the ratio of the values at the first and third quartile of the distribution). An increase in diversity would result in a greater quartile ratio and a decrease would result in a smaller quartile ratio. The quartile ratios for the distributions of normalized motor unit tensions, for the middle roots, are indicated in the upper right of the appropriate panels in Figures 5-7. Quartile ratios for the fast population distributions were 1.7 at early ages, 1.6 at the intermediate ages, and 1.9 at late ages. For the slow population, the quartile ratios were 1.6 at early, 1.7 at intermediate, and 1.7 at late ages. Thus, the quartile ratios change only slightly and without any trend for both the fast and slow populations of motor units. Moreover, when compared using the Kolmogorov-Smirnoff test, none of the distributions were significantly different from one another $(p>0.1)$. Accordingly, we infer that arbor size is unlikely to play a major role during postnatal synapse elimination.

\section{Overlap between middle and extreme root motor units at 4-5 $d$}

Twitch responses were measured from whole ventral roots for 13 normal, early-aged rabhit soleus muscles to determine the degree of overlap of the innervation from the various ventral roots and its dependence on the number of motor units in the extreme (L7 and/or S2) root(s). In all 9 animals where an extreme root contained 18 or fewer soleus motor axons (range, 6-

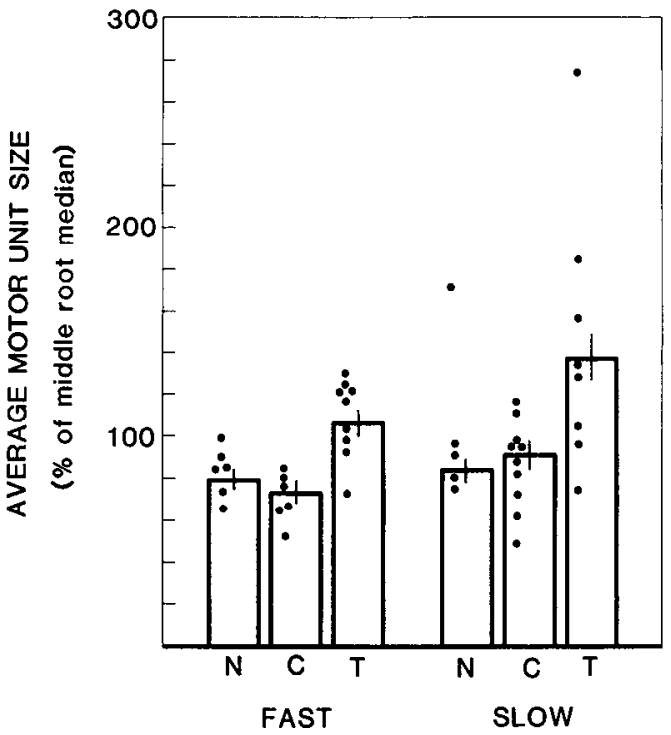

b

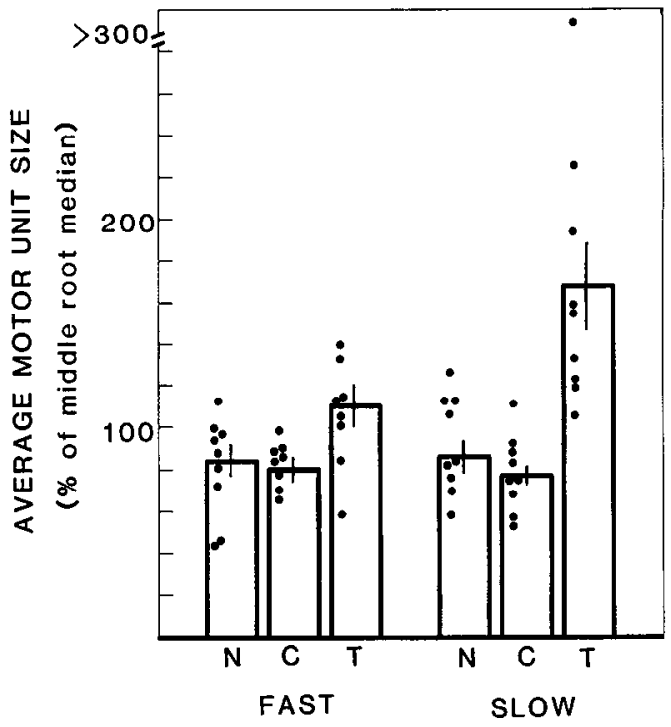

Figure 8. Mean normalized motor unit sizes from normal $(N)$, controlimplanted $(C)$, and TTX-implanted $(T)$ extreme roots of intermediate$(a)$ and late-age $(b)$ animals. Values for fast motor units are shown at the left side of each figure, slow values at right. Bars represent means \pm SEM for pooled units from each group. Dots represent mean sizes of the extreme root motor units from individual animals. For both fast and slow motor units at both ages, control-implanted units are indistinguishable in size from normal units and TTX-implanted units are significantly larger than control-implanted units. Mean values and significance of comparisons are shown in Table 1 . The dot at $172 \%$ for the slow population of intermediate-age normal animals represents the mean value from an animal whose extreme root contributed only one slow unit. Panel $a$ is reproduced, with permission, from Callaway et al. (1987).

18), the whole root twitch tension for S1 was equal to the maximal tension elicited by direct, whole muscle stimulation (within the $3 \%$ error associated with fluctuations in peak tension measurements). This result implies that when an extreme root containing 18 or fewer soleus motor axons was rendered inactive in the TTX experiments described below, virtually all of its synapses were in direct competition with active synapses, and few if any muscle fibers initially lacked active input. 
a

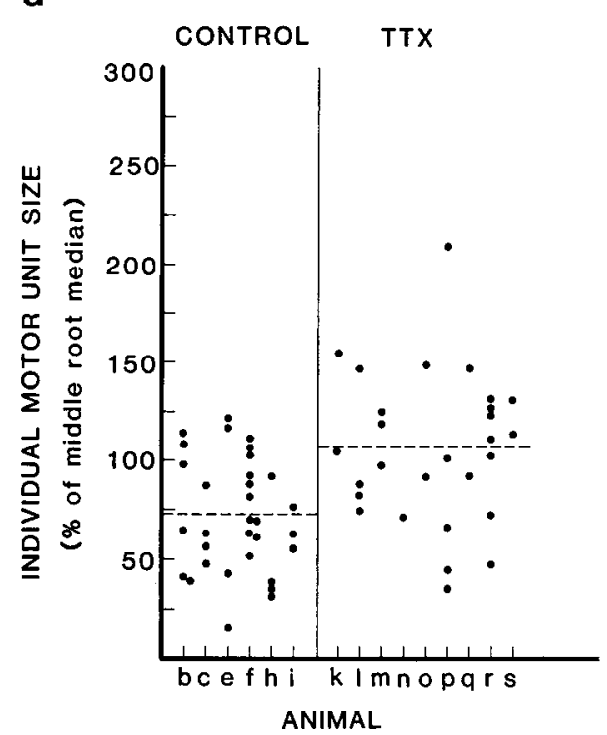

b INTERMEDIATE AGE SLOW UNITS

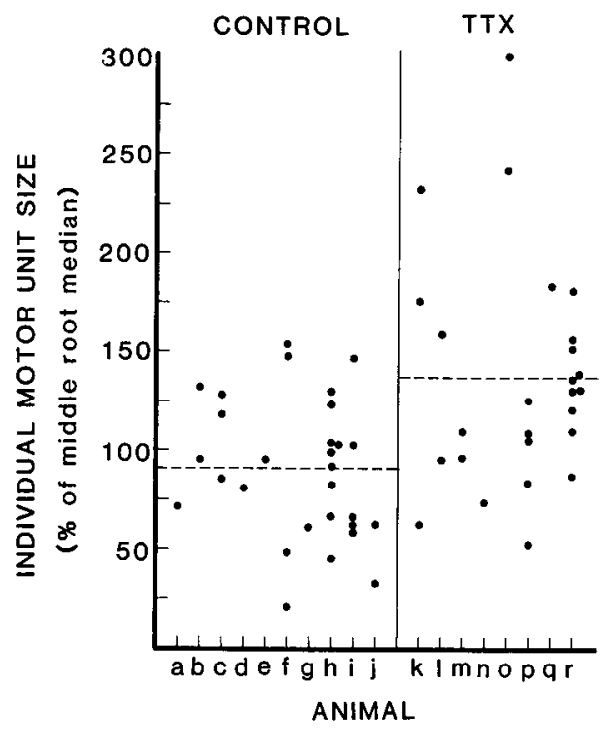

C

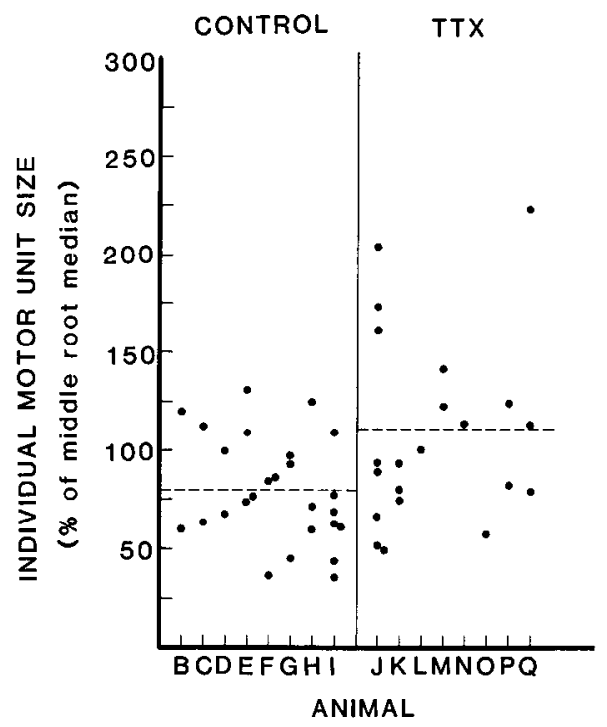

d

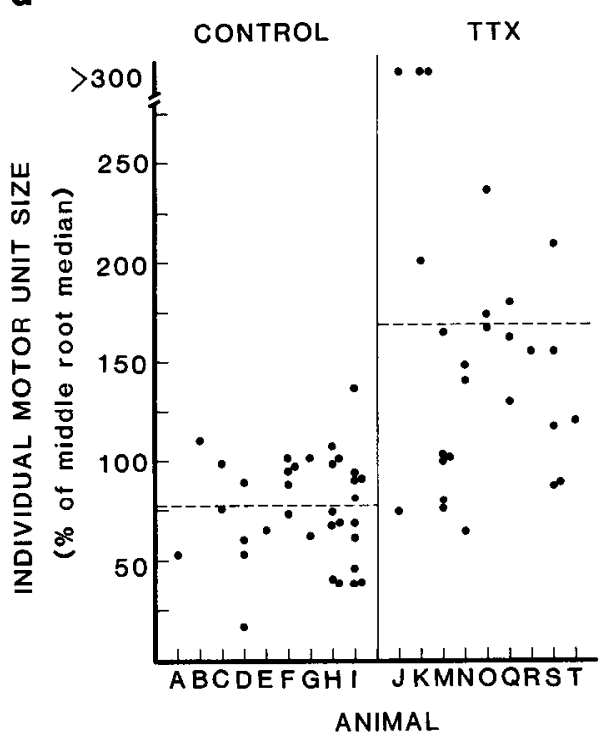

Figure 9. Normalized sizes of individual motor units from extreme roots of control and TTX-implanted animals plotted according to the animals from which they were measured. Mean values for pooled units from each group are represented by dashed lines. Fast units from intermediate age animals are shown in $a$; slow, intermediate age in $b$; fast, late age in $c$; and slow, late age in $d$. Animals are ordered according to the mean percentage tension size of fast motor units from their middle root, with lowest values to the left. Intermediate-age control animals are lettered $a-j$, intermediateage TTX-implanted animals $k-s$, late-age control animals $A-l$, and late-age TTX-implanted animals $J-T$. Panels $a$ and $b$ are reproduced, with permission, from Callaway et al. (1987).

\section{Part II. Effects of differential activity}

\section{Relative motor unit size estimates}

Our principal findings are illustrated in Figure 8, which shows bar graphs of pooled motor unit tensions from extreme roots of normal (N), control-implanted (C), and TTX-implanted (T) animals. Solid circles show the mean normalized tension for individual animals, while the bars show the means $( \pm$ SEM) of the pooled distributions.

First, consider the comparison between control-implanted animals and normal animals. There is no significant difference in the normalized motor unit sizes between normal and controlimplanted, extreme units for either the fast or slow population at either the intermediate or late age $(p>0.2$ for all 4 relevant comparisons; see Table $2 \mathrm{~A}$, rows 8 vs 10 and 16 vs 18 ). Thus, the implantation of control plugs into extreme roots had no discernible effect on synapse elimination. Therefore, any significant difference in size between TTX-implanted and controlimplanted motor unit sizes for any of these groups should be attributable to inactivity induced by the TTX implant.

In contrast to the control implants, Figure 8 shows that the TTX implants had a major effect on motor unit size. At both intermediate and late ages, and for both fast and slow populations, the normalized size of motor units rendered inactive by TTX implantation was significantly larger than that of extreme units from both normal and control-implanted animals. In par- 
ticular, for intermediate-aged TTX-implanted animals, normalized motor unit sizes from extreme (inactivated) roots were half again larger (45-50\%) than the corresponding active, extreme units from control-implanted animals for both the fast population (106 vs $73 \%, p<0.001$; Fig. $8 a$; Table $2,12 \mathrm{~A}$ vs $10 \mathrm{~A}$ ) and the slow population (137 vs $91 \%, p<0.001$; Fig. $8 a$; Table 2, 12A vs 10A). A significant difference was also evident for the late-aged animals. The inactivated motor units werc about one-third larger (38\%) than the control values for the fast population ( 110 vs $80 \%, p<0.02$; Fig. $8 b$; Table $2,20 \mathrm{~A}$ vs $18 \mathrm{~A})$ and more than double the size for the slow population (168 vs $77 \%, p<0.001$; Fig. $8 b$; Table $2,20 \mathrm{~A}$ vs $18 \mathrm{~A}$ ).

We assessed the consistency of the effect across individuals by comparing the mean values from the extreme root(s) of individual animals to the overall mean of pooled values from control animals. The mean size from each TTX-implanted animal is larger than the mean size of pooled, control-implanted units in all but 2 cases. The exceptions were (1) both fast and slow units of one animal from the intermediate group whose extreme (TTX-implanted) root contributed only 1 slow and 1 fast motor unit, and (2) fast units from one animal from the late group whose extreme (TTX-implanted) root contributed only 1 fast motor unit (cf. Fig. 9, animals $n$ and $O$ ). These exceptions are not surprising given the severalfold range of normalized motor unit sizes at the time of the implant (Fig. 5) and the small numbers of motor units from the extreme roots of these animals; thus, we attribute them to statistical fluctuation. Hence, despite some scatter in the experimental data, our results suggest that the TTX treatment had a consistent effect in each animal subjected to a sustained impulse blockade.

Another important issue is whether the TTX blockade affected all or just a special subpopulation of the motor units within the implanted root. To address this, Figure $9 a-d$ plots the size of each motor unit from the implanted, extreme roots of TTX-and control-implanted animals according to the animal from which they were measured. The mean sizes of pooled motor units from each group are indicated by the dashed lines. The individual motor units cover a wide range of sizes for every group, but sizes are shifted toward larger values for the TTXversus control-implanted units in all 4 groups (fast and slow units at both intermediate and late ages). In the TTX-implanted groups, there is not only a higher incidence of relatively large motor units, but a lower incidence of relatively small motor units. This result is consistent with the hypothesis that every motor unit from the implanted root was affected by the TTX treatment.

\section{Differential competition versus delayed synapse elimination}

Thus far, our analysis has demonstrated a highly significant effect of TTX blockade on the relative size of motor units from blocked and normal roots from the same animals. We can envision 2 general explanations for such an effect.

1. The competition hypothesis. Differential activity might confer a competitive advantage on inactive synapses. Consequently, inactive motor units (the winners) would end up larger than normal, and active motor units (the losers) would end up smaller. By the pure form of this hypothesis, the total rate of synapse elimination would be unaffected, and single innervation of the muscle would be achieved over the normal time course.

2. The delay hypothesis. Differential activity might delay the process of synapse elimination on those fibers occupied by a mixture of inactive and active synapses. Consequently, both inactive and active motor units would lose synapses at a slower rate than normal (although not necessarily to the same degree), and both inactive and active motor units would be larger than normal as long as polyinnervation persisted. In addition, the achievement of single innervation would be delayed. Again considering the pure form of this hypothesis, differential activity would not affect the ultimate outcome in terms of which particular synapses were eliminated, only the rate. We used 3 independent approaches to distinguish between these hypotheses.

Our first approach was to determine what effect the TTX blockade had on the absolute size of motor units from the middle roots, as assessed by percentage tension measurements. We found that the mean percentage tension from the middle root was consistently smaller for the TTX animals than for either the control or the normal animals, a result that supports the competition hypothesis. For example, the mean size of slow motor units from intermediate-aged TTX-implanted animals was $16 \%$ smaller than that from normal animals $(1.6 \pm 0.2$ vs $1.9 \pm$ $0.3 \%$; Table $2,11 \mathrm{~B}$ vs $5 \mathrm{~B}$ ) and $30 \%$ smaller than that from control animals $(2.3 \pm 0.3 \%$; Table $2,9 \mathrm{~B})$. Similar results were found for all of the other 6 comparisons, involving fast motor units at the intermediate age and both types at the late age. On average, middle root motor units were $17 \%$ smaller in the TTX cases than in normal and control muscles. This difference is highly significant ( $p=0.001$, Mann-Whitney $U$ test based on individual animal means normalized to the overall mean for normal and control animals combined).

Although these differences were small, they actually exceed the amount predicted on the basis of the numbers of soleus motor axons in extreme and middle roots and the magnitude of the inactivity effects on the extreme roots. On average, the TTX blockade involved fewer than $10 \%$ of the total number of soleus motor axons. Hence, if the inactive motor units ended up about $60 \%$ larger than normal (see above), then by the pure competition hypothesis, the active motor units should end up less than $6 \%$ reduced in size. The fact that our measurements yielded a difference of $17 \%$ could reflect a contribution from individual variability in percentage tension values. An alternative possibility is an actual acceleration of the overall rate of synapse elimination, in addition to a competitive advantage for inactive synapses.

Our second basis for distinguishing between the competition and delay hypotheses was an assessment of the degree of polyinnervation in late age control and TTX cases. If the size difference between motor units from control-implanted and TTXimplanted roots were due to delayed synapse elimination in the TTX-implanted animals, there would be greatcr polyinnervation in their muscles. We used a histochemical silver-staining technique to assess the degree of polyinnervation in muscles from late age TTX- and control-implanted animals (see Materials and Methods). A few polyinnervated fibers were encountered both in control cases ( 2 definite and 1 questionable out of 900 endplates examined in 9 muscles) and TTX cases (4 definite and 1 questionable out of 1100 endplates in 11 muscles). We conclude that fewer than $0.5 \%$ of the fibers were multiply innervated and that there was no significant difference between the 2 groups.

We estimated how much of an increase in polyinnervation in the TTX-implanted muscles would be necessary in order for the delay hypothesis to account for the observed difference in normalized motor unit size between control- and TTX-implanted roots. At the late age, motor units from TTX-implanted roots 
were about $80 \%$ larger than control-implanted (average of 118 and $38 \%$ for the slow and fast populations). Only about $6 \%$ of the motor axons were blocked, however (4.4 on average out of an estimated 70 soleus motor axons). The product of these 2 numbers $(6$ and $80 \%)$ is about $5 \%$, which represents the incidence of polyinnervation predicted by the delay hypothesis. This is an order of magnitude greater than the observed incidence, which argues strongly against the delay hypothesis.

Another measure of polyinnervation is tension overlap. The greater the degree of polyinnervation, the more overlap among motor units. We assessed the overlap between middle and extreme roots in normal and control versus TTX-implanted animals by calculating the percentage of muscle fibers innervated by the extreme root that were not in addition innervated by the middle root. By this measure, termed percentage exclusive innervation, larger values correspond to less overlap (see Callaway et al., 1987, for further details). We have concentrated on the fibers innervated by the extreme root because these are the fibers at which elimination should be slowed in TTX-implanted animals according to the delay hypothesis. At the intermediate age, the percentage exclusive innervation was $34 \pm 8 \%$ (mean \pm SEM) for TTX-implanted versus $19 \pm 8 \%$ for normal and control animals, a difference that was statistically significant ( $p$ $<0.025$, Mann-Whitncy $U$ tcst). This rcsult supports the competition hypothesis and argues against the delay hypothesis. For late age animals, the amount of overlap was quite small. Percentage exclusive innervation was $97-100 \%$ for muscles from both TTX-and control-implanted animals. The slight variations are attributable to error from noise in our measurements, and the results therefore indicate that residual polyinnervation was minimal at this stage, in agreement with the silver-stain results.

\section{Percentage of synapses lost by active and inactive motor units}

Our evidence indicates that the TTX treatment slowed the rate of synapse elimination among motor neurons from the implanted root but did not halt the process altogether. To estimate the change in rate of synapse elimination, we compared percentage tension values at different ages. The decline in motor unit size by fast, inactive, TTX-implanted motor neurons from intermediate-aged animals was only $27 \%(6.7-4.9 \%$; Table 2 , $4 \mathrm{~B}$ vs 12B) compared with $42 \%$ from both normal and control-implanted extreme roots of the same age (Table 2, 4B vs $8 \mathrm{~B}$ and $10 \mathrm{~B})$. This suggests that the TTX blockade reduced the rate of synapse elimination by about one-third (36\%) among inactive motor neurons. For the slow population, the decline in motor unit size for TTX-implanted motor neurons from intermediateaged animals was $45 \%$ (Table $2,4 \mathrm{~B}$ vs $12 \mathrm{~B}$ ) compared with 58 and $53 \%$ for same-aged normal and control-implanted extreme roots respectively (Table $2,4 \mathrm{~B}$ vs $8 \mathrm{~B}$ and $10 \mathrm{~B}$ ). This corresponds to a $22 \%$ reduction in the rate of elimination among inactive motor neurons.

The rate of synapse elimination once the TTX blockade wore off can be estimated by comparing motor unit sizes for TTXimplanted roots examined at intermediate and late ages. For the slow population, average motor unit size declined by $48 \%$ for TTX-implanted roots between intermediate and late ages (Table 2, $12 \mathrm{~B}$ vs $20 \mathrm{~B}$ ). Similar declines took place for normal and control extreme roots (46 and $64 \%$, respectively; Table $2,8 \mathrm{~B}$ vs $16 \mathrm{~B}$ and $10 \mathrm{~B}$ vs $18 \mathrm{~B})$. For the fast population, the decline was $45 \%$ for TTX-implanted roots (Table 2, $12 \mathrm{~B}$ vs $20 \mathrm{~B}$ ) and 36 and $31 \%$, respectively, for normal and control extreme roots (Table $2,8 \mathrm{~B}$ vs $16 \mathrm{~B}$ and $10 \mathrm{~B}$ vs $18 \mathrm{~B}$ ). Although this suggests a slightly greater rate of elimination for fast TTX-implanted neurons while they were active, there is no significant difference between the more sensitive percentage of median values for the fast populations of TTX-implanted motor units at intermediate versus late ages (106 vs $110 \%$ of median, $p>0.2$; Table $1,12 \mathrm{~A}$ vs 20A).

\section{Possibility of repetitive muscle fiber contraction}

We were concerned whether the increased tension produced by previously inactivated motor units might have been an artifactual consequence of repetitive firing (see Ribchester, 1988a). Ribchester and Taxt (1983) reported that endplate potentials at muscle fibers lacking active inputs tend to be prolonged, and the inputs to such fibers can fire repetitively. Either of these effects could lead to repeated contraction of the muscle fiber, resulting in greater than normal tension during a twitch response. They observed that for inactive motor units in muscles with the majority of the fibers inactive, the twitch rise times were slower than normal, apparently due to repeated contraction of muscle fibers. Neither repetitive firing nor prolonged endplate potentials were reported at muscle fibers with even small amounts of active input. These phenomena are therefore unlikely to occur in our TTX-implanted animals, since virtually every muscle fiber had at lcast onc active input at the outset of the cxpcriment, and in the late age animals, all of the inputs had been active for at least $6 \mathrm{~d}$ at the time of the assay. Also, if muscle fibers had fired repeatedly during twitch responses for the TTX-implanted motor units, their rise times would be expected to be slower than normal (as observed by Ribchester and Taxt, 1983). In actuality, twitch rise times for TTX-implanted motor units were slightly faster than for normal or control-implanted motor units (data not shown). It is therefore unlikely that repetitive contraction of muscle fibers contributed to the increased size of twitch responses from inactivated motor neurons in the TTXimplanted animals considered above. For major block animals, however, this is a stronger possibility (see below).

\section{$T T X$-implanted, major block animals}

For 3 TTX-implanted animals analyzed at the intermediate ages, an unusually large fraction of the soleus motor units was inactivated (see Table 1), either because of a branch splitting from the $S 1$ spinal nerve and joining $S 2$ proximal to the TTX implant ( 2 cases) or because of an unusually large number of soleus motor axons in $\mathrm{S} 2$ proper (1 case). In these cases, a substantial percentage of the soleus muscle fibers had all of their inputs inactivated. Based on previous studies, synapse elimination should be delayed in these totally inactive fibers (Thompson et al., 1979; Brown et al., 1981; Duxson, 1982; Callaway and Van Essen, 1989). If muscle inactivity had no other effects, synapse elimination would be expected to proceed in the remaining fibers, with active synapses at a disadvantage when competing with inactive ones. By this logic, inactive motor units should be larger than normal, and active motor units should be the same as or smaller than normal. The actual results from these animals suggest that this is indeed the case for the slow motor units but, surprisingly, not for the fast population.

The twitch responses from combined stimulation of all blocked axons were $80-95 \%$ of maximal direct tension. The responses from active roots were estimated to be $50-90 \%$. From the relative sizes of these measures, we estimate that $50-70 \%$ of the soleus motor axons were inactivated. About $20-50 \%$ of the muscle fibers had no active inputs; $5-20 \%$ had only active inputs, 
and the remainder (45-60\%) had overlapping active and inactive inputs.

For the fast population, the TTX blockade led to an increase in motor unit size for active as well as inactive motor units. Specifically, average motor unit size was $7.4 \pm 1.1 \%$ of maximum for active, fast motor units and $6.6 \pm 0.4 \%$ for inactive, fast units, both of which are significantly larger than middle root motor units from normal, intermediate-aged animals (5.0 \pm $0.5 \%, p<0.025$ ).

For the slow population, the TTX blockade resulted in inactive motor units that were twice as large as normal $(3.8 \pm 0.7$ vs $1.9 \pm 0.3 \%, p<0.01)$. The active motor units, on the other hand, were not significantly larger than normal $(2.6 \pm 0.6 \% \mathrm{vs}$ $1.9 \pm 0.3 \%, p>0.1)$. The difference between active and inactive units was not significant when based on the combined means from all 3 animals. However, there is clearly a significant difference between active and inactive motor units when comparing the intra-animal distributions in 2 of 3 cases: $5.2 \pm 0.4 \%$ (19 units) vs $3.5 \pm 0.3 \%$ (4 units), $p<0.05$; and $3.1 \pm 0.3 \%$ (14 units) vs $1.4 \pm 0.4 \%$ (13 units), $p<0.001$. Therefore, it appears that the fast and slow populations were affected differently by the activity block of the same percentage of motor axons; the results from the fast population were similar to those expected from activity block of the entire neuronal population, while the results from the slow population were more similar to the results following blockage of a minor root.

\section{Discussion}

In this study we have tested for (1) a correlation between axonal arbor size and the number of synapses lost by motor neurons of a given contractile type; (2) differences in the degree of polyinnervation and rate of synapse elimination for fast versus slow muscle fibers; (3) spatial gradients in the extent of synapse loss for motor neurons from different spinal positions; and (4) differences in competitive ability between active and inactive motor neurons. Our major findings relate to the last 2 issues, but we will start by dealing expeditiously with points 1 and 2 .

\section{Interactions during normal maturation}

\section{Axonal arbor size}

Previous reports have noted an initial increase in diversity of motor unit sizes during the first 2 postnatal weeks and have suggested accordingly that terminals belonging to large motor units might be at a competitive advantage over those from smaller motor units (Gordon and Van Essen, 1981; Van Essen, 1982). On the other hand, it has been suggested that larger motor units might be at a disadvantage owing to the metabolic constraints of maintaining a larger axonal arbor (Smalheiser and Crain, 1984). Our results do not support cither hypothesis. There were only small differences between distributions of middle motor unit sizes for animals of different ages based on their quartile ratios; and the distributions do not vary significantly with age. Thus, we found no indication that arbor size influences the competitive ability of motor neurons during synapse elimination in the rabbit soleus.

The difference between the present results and those from previous studies is most likely due to our having separated fast and slow motor unit populations in this study. Without this separation, our results also show an increase in diversity, since fast units averaged only 1.9 times larger than slow at early ages, but the difference increased to 3.1 times at late ages. As discussed below, this change in relative size probably represents, at least to some degree, a difference in rate of synapse elimination for fast versus slow muscle fibers.

\section{Fast versus slow muscle fibers}

Our results are consistent with the suggestion of Gordon (1984) that the extent of polyinnervation at birth is greater for the slow population of rabbit soleus muscle fibers than the fast population. Both sets of results suggest either that fast fibers are initially contacted by fewer motor neurons than are slow fibers or that synapse elimination begins earlier for the fast population. However, this interpretation is dependent on the assumption that the ratio of the tension generated by individual fast and slow muscle fibers does not change substantially from birth to $15 \mathrm{~d}$. Gordon (1984) found no change during this period in the ratio of cross-sectional areas for Type I (presumed slow) and Type II (presumed fast) fibers. If the ratio of specific tensions (force/ $\mu \mathrm{m}^{2}$ ) also remains constant, then the ratio between average motor unit size in polyinnervated muscles to that in singly innervated (late age) muscles will reflect the number of synapses per muscle fiber. If this assumption is incorrect, this ratio will reflect a combination of polyinnervation and changes in muscle characteristics. That our calculations are not far off the mark is supported by the good agreement between our estimated value of 1.75 synapses per muscle fiber at intermediate ages (average for fast and slow) and the estimate of 1.7 synapses per fiber obtained from intracellular recording in rabbit soleus muscles of the same age (Soha et al., 1987).

Based on the present evidence, the rate of synapse elimination appears to be greater for the slow than for the fast population of muscle fibers, but with both populations reaching the singly innervated state at about the same time. Soha et al. (1987) reached a similar conclusion about the synchrony of onset of single innervation in fast and slow fibers.

\section{Rostral versus caudal motor neurons}

Miyata and Yoshioka (1980) suggested on the basis of an indirect assay, that rostral motor neurons lose many more synapses than caudal motor neurons in the neonatal rat soleus. Subsequent direct measurements of motor unit sizes have revealed no rostral versus caudal bias for the soleus muscle in the rat (Gordon and Van Essen, 1983; Thompson, 1983b), mouse (Fladby, 1987), or the rabbit (Gordon and Van Essen, 1983). Our results support and extend these findings, in that we do not find any marked difference in the extent of synapse elimination for either fast or slow motor neurons from the rostral versus caudal extremes in the rabbit soleus motor pool.

\section{Extreme versus middle motor neurons}

Unexpectedly, we found that during normal maturation, motor unit tensions decline more extensively for inputs from extreme spinal roots than from the middle root. This difference is small (ca. 20-25\%) but highly significant for both the fast and slow populations of motor neurons and for both rostral and caudal extremes; and it develops during a brief window between early (4-5 d) and intermediate (8-9 d) ages. Conceivably, it might reflect a difference in tension generated per fiber among muscle fibers innervated by the extreme versus middle root, rather than a difference in relative number of synapses lost. Such a change might result from (1) selective loss of terminals from fibers according to contractile strength, (2) selective loss at fibers whose position in the muscle does not allow them to contribute effectively to the measured tension, (3) a change in contractile strength 
of fibers according to the neurons that innervate them, or (4) a mechanical artifact in which fibers innervated through extreme roots are less effective in contributing to muscle tension (except in the early age animals), perhaps because they lie in a particular region of the muscle where the length/tension relationship might be different from the bulk of the muscle. The first 3 possibilities all seem quite unlikely given the degree of overlap between extreme and middle root innervation at early ages (complete overlap) and intermediate ages (about 80\% overlap for fibers innervated by the extreme root) coupled with the observation that extreme units generate at least as much tension as middle units at the early age. The fourth possibility is hard to evaluate because it is not known whether there is topography of rabbit soleus muscle innervation. However, it also seems unlikely because it implies an age-dependent change involving regions innervated by both rostral and caudal extremes. Hence, we favor the interpretation that there is an actual difference in synapse loss between extreme and middle roots.

We can envision 2 types of mechanism that might account for differential synapse elimination according to spinal position. The first involves activity-dependent effects on motor unit size. Based on our finding that inactivity increases a motor neuron's competitive ability, higher activity levels might be expected to confer a disadvantage. To account for our results, extreme motor neurons would have to be more active than those in the middle. No direct evidence on this point is available, but it is conceivable that such differences might result from variations in afferent connections to extreme and middle motor neurons or from variations in cell body size.

The second possibility, which is more appealing to us, involves chemospecific matching between motor neurons and muscle fibers. Suppose, for example, that there were positional differences in the spinal cord, such that soleus muscle fibers were better matched to neurons from the middle of the soleus motor pool than to those from either extreme, and that this differential matching were manifested during synapse elimination rather than during synapse formation. Alternatively, there might be gradients in the muscle as well as in the motor neuron pool. For instance, each motor neuron might be optimally matched to muscle fibers in a restricted portion of the muscle, with the rostrocaudal spinal axis mapping onto a particular axis (say, dorsoventral) in the muscle. If the gradient in the muscle were shallower than the gradient in the spinal cord, then motor neurons from extreme positions would be optimally matched with fewer muscle fibers than motor neurons from middle positions. Again supposing that the differential matching were manifested during synapse elimination, this would lead to greater synapse loss by extreme motor neurons.

Evidence for some form of segmental specificity between nerve and muscle has come from reinnervation experiments, in which intercostal muscles are preferentially reinnervated by preganglionic autonomic neurons from specific spinal segments (Wigston and Sanes, 1982, 1985) or in which a topographic map is restored by reinnervating spinal motor ncurons (Laskowski and Sanes, 1987). It is not clear from these studies, however, whether the modest segmental biases revealed at the time of the assay reflect a difference in synapse formation or in synapse elimination. More direct evidence for a role of differential synapse elimination in the sharpening of topography has come from studies of segmental innervation patterns during normal maturation in the gluteus and lateral gastrocnemius muscles of rats (Brown and Booth, 1983; Bennett and Lavidis, 1984; Bennett et al., 1986; Bennett and Ho, 1988). These observations suggest that the synapses from a single motor neuron initially innervate a large but still crudely appropriate topographic area, and as synapse elimination proceeds the more topographically appropriate connections tend to win the competitions on the individual muscle fibers. Similar observations were reported by Lichtman and Purves (1980) in the superior cervical ganglion of the hamster. On the other hand, the soleus muscle apparently lacks segmental topography in both mouse (Fladby, 1987) and rat (Thompson et al., 1984); it is unclear what the pattern is in the rabbit, which has many times more muscle fibers. Thus, while the aforementioned findings are supportive of the matching hypothesis, they are consistent with either the single-gradient or double-gradient version.

\section{Activity}

Competition between active and inactive synapses

We have tested the influence of activity on the ability of neuromuscular synapses to compete for occupancy of endplates during neonatal synapse elimination. In the paradigm used, active and inactive synapses were pitted directly against one another, at endplates on active muscle fibers, by inactivating a small fraction of the soleus motor axons. There were 4 key findings from these experiments. (1) Motor unit tensions from TTX-inactivated roots were approximately half again larger than those from control-implanted or normal extreme roots. (2) This effect appeared over a period of 4-5 d of differential activity, and it persisted even when activity in the initially inactive root was restored. (3) The increase in average tension from inactive motor units was coupled with a small but significant decrease in avcrage tension of motor units from the active middle root. (4) There was no discernible increase in the incidence of polyinnervation as assessed by physiological tension overlap measurements or by histological analysis of silver-stained terminals.

In interpreting these observations, we have considered a variety of potential sources of error or systematic bias in the degree to which our twitch tension assay reflects actual motor unit sizes. Our findings are not attributable to repetitive firing of muscle fibers or differences in the underrepresentation of maximal twitch responses for different muscles. Since inactivated motor units remained larger than normal even in singly innervated late-age muscles, it also is highly unlikely that the TTX treatment simply caused a delay in synapse elimination among muscles fibers innervated by the implanted root or that the larger relative motor unit size was an artifact from unmasking of normally subthreshold connections (or a higher incidence of subthreshold inputs in the active population).

We conclude that differential activity had a direct effect on synaptic competition, leading to a lower rate of synapse loss among inactive motor neurons, which was offset by an increased rate of synapse loss among active motor neurons. However, this increased competitiveness was provided to motor neurons that initially were at a slight disadvantage owing to their origin from an extreme spinal root. For slow motor units, the effect was large enough that the inactive units actually became significantly larger than the active units from the middle root $(p<0.01$ for intermediate and $p<0.005$ for late-age animals, Mann-Whitney $U$ test). For fast motor units, the effect was only sufficient to restore the extreme motor units to approximate parity with the middle root units.

There are 2 plausible explanations that might account for the greater effect of differential activity on slow motor units. First, 
the overall rate of synapse elimination was greater for slow than for fast motor units ( 58 vs $42 \%$ of synapses lost for extreme roots between early and intermediate ages; see Table 2). If differential activity elicited a fractional change rather than an absolute rate change, then a greater effect on slow motor units would be predicted. Second, average activity levels are reportedly about twice as great for slow as for fast motor units (Navarette and Vrbova, 1983). Consequently, TTX-inactivation would lead to a greater change in activity for the slow motor units, which in turn might have a greater effect on motor unit size.

Five studies have reported evidence suggesting an advantage to more active synapses, which contrasts sharply with the conclusions of the present results. One of these studies involved differential activity during normal neuromuscular development (Ridge and Betz, 1984); 3 involved reinnervation of adult muscle (Ribchester and Taxt, 1983, 1984; Ribchester, 1988b); and the last involved a tissue culture preparation (Magchielse and Meeter, 1986).

The closest paradigm to our own was the study of Ridge and Betz (1984), who used electrical stimulation to increase the activity of 1 of the 2 nerves innervating the lumbrical muscle in neonatal rats. They reported that motor units from stimulated nerves were slightly larger than those from unstimulated nerves. However, these differences were at best marginally significant when tested by standard procedures (Student's $t$ test or MannWhitney $U$ test). They claimed that the result was significant when analyzed by another procedure, but the statistical validity of this alternate procedure is questionable in our opinion. ${ }^{1}$ While we are not convinced by their arguments, it is of course possible that their interpretation is nonetheless correct. If so, the difference between their results and ours might be related to factors such as the specics uscd (rat vs rabbit), the particular muscle (lumbrical vs soleus), or to the means of achieving differential activity (high vs normal activity in their experiments; normal activity vs inactivity in ours).

One noteworthy difference between development in the rat lumbrical and rabbit soleus muscles is that the number of muscle fibers in the lumbrical nearly doubles during the period of synapse elimination (Betz el al., 1979), while there is a constant number for the rabbit soleus (Bixby and Van Essen, 1979). Thus, during the period when Ridge and Betz (1984) invoked differential activity (during the second postnatal week), there is normally no net loss of synapses; rather, each lumbrical motor neuron maintains a roughly constant number of synapses because synapse loss is offset by formation of synapses on the newly generated fibers (Betz et al., 1979). This suggests that activity could be an advantage for synapse formation but a disadvantage during synapse elimination, which would be consistent with both the present results and those of Ridge and Betz (1984). By this hypothesis, there would be little net effect of

\footnotetext{
In their published report, motor units whose sizes were expected to differ from normal (from the "minority" populations mentioned above) were compared with all the other units from the same animal (including the contralateral muscle), whose sizes were expected to be relatively normal. Thus, numerous ratios were obtained from a small sample of "minority" units; for example, the 4 units from the animals in which a minority of the motor neurons were stimulated yielded 23 ratios. The pooled ratios for units expected to be larger than normal were then compared with ratios expected to be smaller. The result was deemed significant based on statistical tests on the pooled ratios ( $U$ test or $t$ test), apparently without regard for the need for samples in such tests to be obtained independently. Because the numerous ratios obtained in the normalization procedure were dependent on a relatively small number of samples, we doubt that their statistical analysis was valid.
}

differential activity on motor unit size during early stages of synapse elimination in the lumbrical (as Ridge and Betz observed) because of the offsetting differences in the amount of synapse formation and elimination; but there would be a relative increase in size for inactive motor units in the rabbit soleus (present result). If the differential activity in the lumbrical were maintained until the entire muscle was singly innervated, this hypothesis predicts that the stimulated motor units would end up smaller in size relative to the unstimulated units.

Three studies (Ribchester and Taxt, 1983, 1984; Ribchester, 1988 b) have examined the effect of inactivity on the regeneration of connections in the lumbrical muscle of adult rats following partial or complete denervation. They found that nerve inactivity reduced the eventual size of regenerated motor units whether in competition with normally active regenerating axons (Ribchester and Taxt, 1983) or in competition with collateral sprouts from active, intact motor axons (Ribchester, 1988b). They also found that active motor axons were more successful in reinnervation when competing with sprouts of inactive motor axons than with active ones (Ribchester and Taxt, 1984).

These findings clearly demonstrate a net advantage to active motor neurons in reinnervation of adult rat muscle. In order to reconcile this with our own findings, one can again invoke the fact that the experiments were carried out in different species, different muscles, and at different ages. However, the apparent discrepancy might be related to other aspects of the experimental paradigms. In the reinnervation experiments, differential activity could confer a competitive advantage in several distinct ways. We have already raised the possibility that inactivity might reduce the capacity to form new synapses. In addition, inactivity might reduce the rate of axonal regeneration and/or the ability to respond to sprouting influences. Any of these possibilities would put inactive motor ncurons at a disadvantage in an adult reinnervation paradigm but would have little or no effect on normal postnatal synapse elimination, where axons are not regenerating and there is little tendency for sprouting to occur even under conditions that induce massive sprouting in the adult (Brown et al., 1981).

A related consideration is that the regeneration paradigm involves an inherent asymmetry in the occurrence of sprouting signals. Inactive muscle fibers should continuously release a sprouting signal that would occasionally induce collateral sprouting and synaptic takeover by active motor neurons. In contrast, active muscle fibers no longer release overt sprouting signals and are thus more likely to remain stably innervated. Consequently, any muscle innervated by a mixture of active and inactive neurons will not be in a steady state even when single innervation is achieved. Competition at dually innervated endplates will result in the removal of one input, but if the survivor is an inactive input, the subsequent production of sprouting signal will tend eventually to restore an active input. Thus, there may be a tendency for asymmetric sprouting, involving gradual encroachment by active motor neurons onto inactive muscle fibers even though the probabilities of survival at a dually innervated endplate might initially be equal or even favor the inactive synapse. In principle, this asymmetry also applies to our own paradigm using neonatal muscles, but it is probably much less important there owing to the reduced tendency for sprouting to occur in neonatal muscles.

An in vitro system, in which more active synapses are preferentially retained during elimination, has been described by Magchielse and Meeter (1986). In their system, chick ciliary 

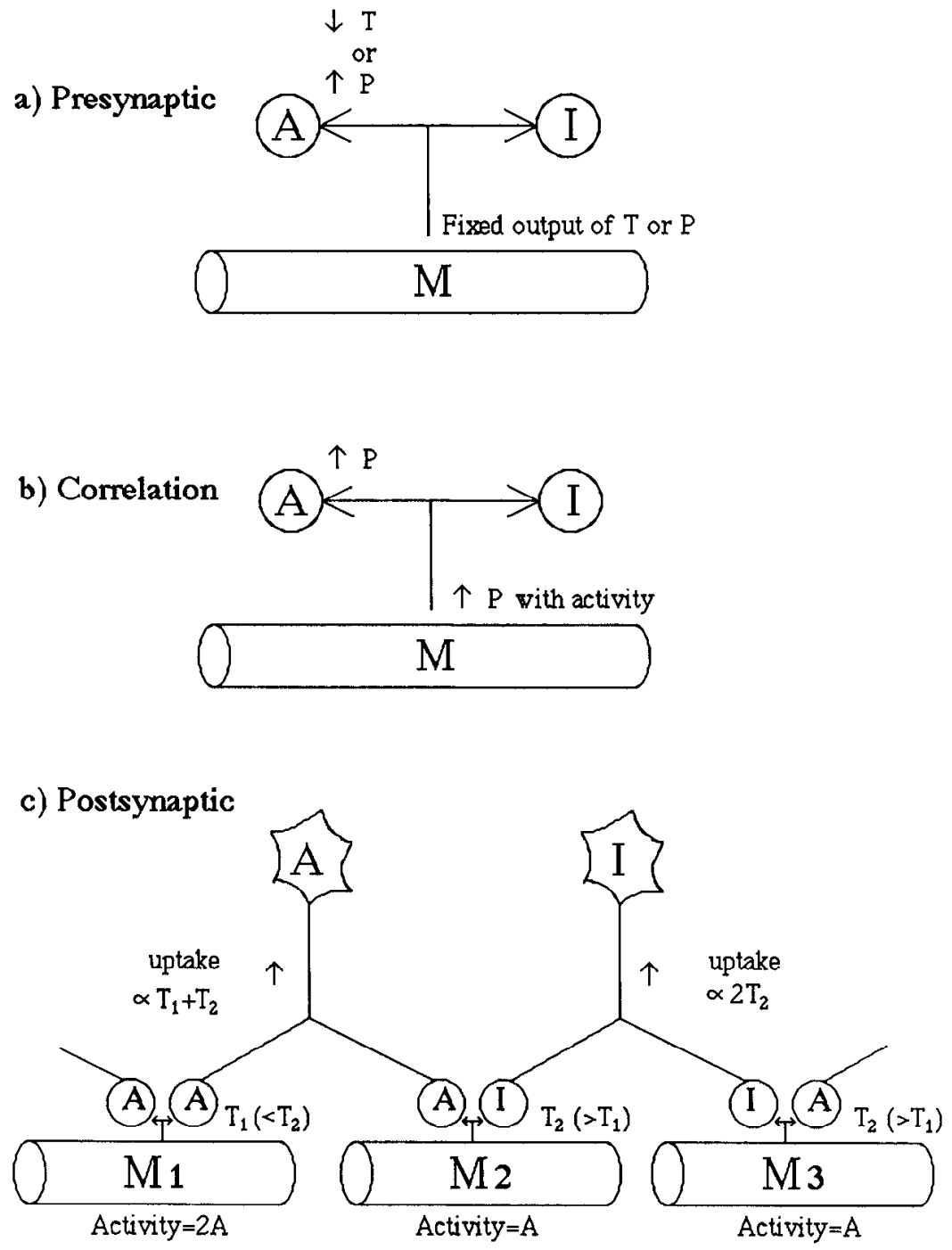

Figure 10. Schematic illustrations of 3 non-Hebbian mechanisms that could convey an advantage to the terminals of less active motor neurons during neuromuscular synapse elimination. $a$, Strictly presynaptic mechanism. The more active nerve terminal (circled $A$ ) incorporates either more trophic factor $(T)$ or less protease $(P)$ than the relatively inactive terminal (circled $I$ ) from a pool of fixed size produced by the muscle fiber $(M) . b$, Mechanism conveying a disadvantage to more active terminals dependent on temporally correlated postsynaptic activity. There is a transient increase in protease release from the muscle fiber when it is activated, and the protease is taken up preferentially by the nerve terminal (circled $A$ ) whose temporally correlated activity initiated the postsynaptic response. $c$, Mechanism based on regulation by a postsynaptic mechanism; presynaptic activity is important only in that it determines the levels of postsynaptic activity at the individual muscle fibers $\left(M_{1,2,3}\right)$. More active motor neurons (e.g., neuron $A$ ) innervate muscle fibers whose average activity is greater than that for the set of muscle fibers innervated by a relatively inactive motor neuron (e.g., neuron $I$ ). Thus, if more active muscle fibers $\left(M_{i}\right)$ produce less trophic substance than less active muscle fibers $\left(M_{2.3} ; T_{1}<T_{2}\right)$, the more active motor neuron will not have access to as much of the trophic substance (per terminal) as a relatively inactive motor neuron. Terminals of the more active motor neuron will therefore be at a disadvantage if their competitive ability depends on the level of trophic factor reaching their parent motor neuron. (parasympathetic) ncurons were made to inncrvate skclctal muscle fibers polyneuronally, but the multiple inputs were distributed across spatially separated endplates. Stimulation-induced elimination of endplates was subsequently observed. When unstimulated synapses shared innervation of a muscle fiber with stimulated synapses, the unstimulated endplates were preferentially removed. The difference between their result and ours may reflect the difference in motor neuron type or possibly the lack of direct competition between synapses sharing a single endplate. These results suggest that synapse elimination might be regulated differently in different neuromuscular systems (e.g., on tonic vs twitch muscle).

\section{Combined pre-and postsynaptic partial inactivity}

The experiments in which nearly half of the soleus motor axons were inactivated by TTX (TTX major block animals) led to several significant findings. First, inactive motor units were larger than normal for both the fast and slow populations in all 3 muscles examined. This is not surprising given that complete nerve inactivation is known to slow down synapse elimination markedly (see Thompson, 1985, for review). A plausible explanation is that inactive muscle fibers release a sprouting or trophic factor (see Brown et al., 1980) that would reduce the natural tendency of neonatal motor neurons to withdraw their terminals.

Unexpectedly, we found in these experiments that active motor units were significantly larger than normal for the fast population, but less so or not at all for the slow population. How might one account for an effect on active motor units that differs for fast and slow types? One possibility is that inactive muscle fibers might release a diffusible factor that reduces the rate of synapse elimination at neighboring endplates. The differential effect would be explained if for any reason the sprouting factor operated more effectively or at a lower threshold on fast terminals compared to slow terminals. Alternatively, there conceivably could be separate factors operating independently for fast and slow populations; the differential effect could arise from greater production of the fast sprouting factor or greater sensitivity to a given concentration. Still another possibility, unrelated to sprouting, is that graded changes in postsynaptic activity might reduce the rate of synapse elimination on fast fibers but not on slow fibers (see Callaway et al., 1989). Although we cannot rule it out, this effect seems unlikely given that we found no discernible change in the overall rate of synapse elimination in our main experiments involving a less extensive activity block (see above). 


\section{Developmental rules and molecular signals mediating competition}

A "Hebbian" synapse is one in which correlated pre- and postsynaptic activity leads to strengthening of the synapse (Hebb, 1949). Our results indicate that the neonatal neuromuscular junction is not Hebbian. However, there are several ways in which this non-Hebbian behavior might be mediated. In one, the correlation between pre- and postsynaptic activity would still be critical, but the sign of the effect would be the opposite of that suggested by Hebb. In another, postsynaptic activity would be irrelevant, with the outcome being determined simply by differences in presynaptic activity levels. The third is a more subtle mechanism, in which postsynaptic activity is the critical factor and the outcome would depend on activity profiles associated with a population of muscle fibers and motor neurons rather than a single endplate.

It is useful to discuss these mechanisms in the context of particular cellular signals that could mediate the critical interactions. A variety of possible signals have been proposed to play an important role during synapse elimination. These include a diffusible trophic factor (Thompson et al., 1979), a stable scaffolding factor in the extracellular matrix (Van Essen, 1982), and a calcium-activated protease associated with either the pre- or postsynaptic elements (O'Brien et al., 1978, 1984; Connold et al., 1986). Figure 10 schematically illustrates the nature of each type of non-Hebbian competition between an active $(A)$ and inactive $(I)$ presynaptic terminal. Plausible mechanisms are also shown that are based on release of a postsynaptic trophic factor $(T)$ and/or protease $(P)$ as the critical molecular signal.

For the strictly presynaptic mechanism (Fig. 10a), suppose that the muscle fiber $(M)$ synthesizes and releases trophic factor or protease at a steady rate. The active terminal would be at a disadvantage relative to its inactive neighbor if activity either decreased the uptake of the trophic factor or increased the uptake of the protease (assuming that the protease has a negative effect on synaptic competitiveness). An alternative presynaptic mechanism is suggested by the observation of Connold et al. (1986) that low calcium and inhibition of protease can slow the rate of synapse elimination. If the relevant protease was activated by calcium and localized to presynaptic terminals, then the greater calcium influx associated with higher activity would result in more protease activity within more active terminals. Again presuming that the greater protease activity would confer a competitive disadvantage, less active terminals would tend to outcompete those with higher activity.

For the correlation mechanism (Fig. 10b), suppose that postsynaptic activity transiently increases the output of protease and that presynaptic activity increases the rate at which extracellular fluid is incorporated, say by vesicular endocytosis. Suppose also, for convenience, that the time courses of these 2 processes are similar. The correlation between pre- and postsynaptic activity would be important in putting the active terminal at a disadvantage, particularly if the baseline accumulation of protease were minimal as a consequence of low protease production or uptake in the inactive state.

For the postsynaptic mechanism (Fig. 10c), suppose that each muscle fiber decreases its output of trophic factor when active and that this factor is taken up at equal rates by both inactive and active terminals. Despite the local symmetry at each endplate, there would be a global asymmetry because those muscle fibers associated with an active motor unit must have an average activity level that is higher than that of muscle fibers associated with an inactive motor unit. Consequently, active motor neurons would receive slightly less trophic factor per synapse; if the competitiveness of each synapse depends in part on the overall level of trophic factor reaching the parent motor neuron, then synapses of the active motor neuron would be at a disadvantage. These are by no means the only plausible mechanisms that might underlie neuromuscular synaptic competition. However, they do illustrate a wide range of possibilities that are consistent with the available data.

\section{Functional significance}

According to the size principle (Henneman and Olson, 1965), the recruitment threshold of a motor neuron is directly related to motor unit size: Smaller motor units are recruited first and progressively larger motor units are recruited as excitation of the motor pool increases. The presumed advantage of this configuration is that force generated by a large muscle can be finely graded when small movements are needed, while maximal force can be generated with a moderate number of motor neurons. How this relationship is established during development is not known. An intriguing possibility is that it results from preferential retention of less active inputs during synapse elimination. If a motor neuron's level of activity at birth is closely related to its adult recruitment threshold, such a role for activity in the regulation of motor unit size would seem quite plausible.

While this is an attractive hypothesis, it surely is not the sole factor regulating motor unit size. For example, activity levels are presumably not rigidly predetermined, and feedback loops could link activity levels with motor neuron size and supply of trophic factor from the periphery. To give an explicit illustration, consider a neuron with low average activity, which accordingly innervates muscle fibers with reduced activity. Suppose, as suggested above, that these fibers produce more than the average amount of trophic factor and that the elevated level of retrogradely transported trophic factor causes the motor neuron soma to grow relatively rapidly. This could lead to still lower activity on average, thereby completing a positive-feedback loop.

Various other interactions and feedback loops might also contribute to the regulation of motor unit size. Our point, however, is not to enumerate all the possibilities, but to emphasize the importance of eventually placing each individual mechanism in the context provided by the concurrent operation of other developmental interactions.

An advantage to more active inputs and/or strengthening of connections with correlated pre- and postsynaptic activity has been demonstrated in other systems - in the development of the visual system and in long-term potentiation in the hippocampus (Kelso et al., 1986; Singer, 1987). In the hippocampus, the sign of the effect is apparently appropriate to the function of the system, given its involvement in learning, the task for which Hebbian synapses were originally hypothesized (Hebb, 1949). In the mammalian visual system (see Singer, 1987, for review), early segregation of inputs from left and right eyes is presumably needed (in some species) as a prelude to the generation of appropriate binocular receptive field properties. A Hebbian rule is presumably advantageous for generating this segregation insofar as the activity of neighboring ganglion cells from the same eye is better correlated than that of corresponding cells from different eyes. Interestingly, the advantage to correlation in this system apparently applies to synchronuos pre- and postsynaptic 
inactivity as well as activity: Inputs from a deprived eye are reportedly at an advantage when postsynaptic responses in the cortex are blocked with the inhibitory agonist muscimol (Reiter and Stryker, 1988). Activity dependence for the segregation of ocular dominance stripes has also been demonstrated in 3-eyed frogs produced by embryonic eye transplants (Reh and Constantine-Paton, 1985). Here, it appears that a mechanism implicated in refinement of topography (Meyer, 1983) can also cause a more dramatic segregation given a higher degree of noncorrelated activity.

A competitive advantage to less active synapses has not to our knowledge been described in any system previously. However, the importance of Hebbian mechanisms in some systems and the elegance of the hypothesis certainly do not mandate the presence of such a mechanism in all activity-regulated neural systems. Hopfield et al. (1983) have demonstrated that in simulated neural networks, synaptic weakening during correlated pre- and postsynaptic activity is useful for the removal of spurious memory states. We suspect that the neuromuscular system may not be the only one in which activity is a disadvantage for retention of connections. Rather, the sign of an activity influence, if any, should relate to the function of the system in question.

\section{References}

Bennett, M. R., and S. Ho (1988) The formation of topographical maps in developing rat gastrocnemius muscle during synapsc climination. J. Physiol. (Lond.) 396: 471-496.

Bennett, M. R., and N. A. Lavidis (1984) Development of the topographical projection of motor neurons to a rat muscle accompanies loss of polyneuronal innervation. J. Neurosci. 4: 2204-2212.

Bennett, M. R., S. Ho, and N. A. Lavidis (1986) Competition between segmental nerves at end-plates in rat gastrocnemius muscle during loss of polyneuronal innervation. J. Physiol. (Lond.) 381: 351-376.

Betz, W. J.. J. H. Caldwell, and R. R. Ribchester (1979) The size of motor units during postnatal development of rat lumbrical muscle. J. Physiol. (Lond.) 297: 463-478.

Betz, W. J., J. H. Caldwell, and R. R. Ribchester (1980) The effects of partial denervation at birth on the development of muscle fibers and motor units in rat lumbrical muscle. J. Physiol. (Lond.) 303: 265279.

Bixby, J. L., and D. C. Van Essen (1979) Regional differences in the timing of synapse elimination in skeletal muscles of the neonatal rabbit. Brain Res. 169: 275-286.

Bixby, J. L., J. H. R. Maunsell, and D. C. Van Essen (1980) Effects of motor unit size on innervation patterns in neonatal mammals. Exp. Neurol. 70: 516-524.

Brown, M. C., and C. M. Booth (1983) Postnatal development of the adult pattern of motor axon distribution in rat muscle. Nature 304 : 741-742.

Brown, M. C., and P. B. C. Matthews (1960) An investigation into the possible existence of polyneuronal innervation of individual skeletal muscle fibres in certain hind-limb muscles of the cat. J. Physiol. (Lond.) 151: 436-457.

Brown, M. C,. J. K. S. Janscn, and D. C. Van Essen (1976) Polyneuronal innervation of skeletal muscle in new-born rats and its elimination during maturation. J. Physiol. (Lond.) 261: 387-422.

Brown, M. C., R. L. Holland, W. G. Hopkins, and R. J. Keynes (1980) An assessment of the spread of the signal for terminal sprouting within and between muscles. Brain Res. 210: 145-151.

Brown, M. C., R. L. Holland, and W. G. Hopkins (1981) Restoration of focal multiple innervation in rat muscles by transmission block during a critical stage of development. J. Physiol. (Lond.) 318: 355364.

Callaway, E. M., and D. C. Van Essen (1989) Slowing of synapse elimination by $\alpha$-bungarotoxin superfusion of the neonatal rabbit soleus muscle. Dev. Biol. 131: 356-365.

Callaway, E. M., J. M. Soha, and D. C. Van Essen (1987) Competition favouring inactive over active motor neurons during synapse elimination. Nature 328: 422-426.

Connold, A. L., J. V. Evers, and G. Vrbova (1986) Effect of low calcium and protease inhibitors on synapse elimination during posinatal development in the rat soleus muscle. Dev. Brain Res. 28: 99-107.

Duxson, M. J. (1982) The effect of postsynaptic block on the development of the neuromuscular junction in postnatal rats. J. Neurocytol. 11: 395-408.

Fladby, T. (1987) Postnatal loss of synaptic terminals in the normal mouse soleus muscle. Acta Physiol. Scand. 129: 229-238.

Fladby, T., and J. K. S. Jansen (1987) Postnatal loss of synaptic terminals in the partially denervated mouse soleus muscle. Acta Physiol. Scand. 129: 239-246.

Gordon, H. (1984) Postnatal development of motor units in rabbit and rat soleus muscles. Ph.D. dissertation, California Institute of Technology.

Gordon, H., and D. C. Van Essen (1981) Motor units diversify in size as synapse elimination proceeds in the neonatal rabbit soleus muscle. Soc. Neurosci. Abstr. 7: 179.

Gordon, H., and D. C. Van Essen (1983) The relation of neuromuscular synapse elimination to spinal position of rabbit and rat soleus motoneurones. J. Physiol. (Lond.) 339: 591-597.

Gordon, H., and D. C. Van Essen (1985) Specific innervation of muscle fiber types in a developmentally polyinnervated muscle. Dev. Biol. 111: $42-50$.

Hebb, D. O. (1949) The Organization of Behaviour. A Neuropsychological Theory, 5th ed., p. 62, Chapman and Hall, London.

Henneman, E., and C. B. Olson (1965) Relations between structure and function in the design of skeletal muscles. J. Neurophysiol. 28: 581-598.

Hopfield, J. J., D. I. Feinstein, and R. G. Palmer (1983) 'Unlearning' has a stabilizing effect in collective memories. Nature 304: 158-159.

Hopkins, W. G., M. C. Brown, and R. J. Keynes (1985) Postnatal growth of motor nerve terminals in muscles of the mouse. J. Neurocytol. 14: 525-540.

Karnovsky, M. J., and L. Roots (1964) A 'direct colouring' thiocholine method for cholinesterase. J. Histochem. Cytochem. 12:219-221.

Kelso, S. R., A. H. Ganong, and T. H. Brown (1986) Hebbian synapses in hippocampus. Proc. Natl. Acad. Sci. USA 83: 5326-5330.

Laskowski, M. B., and J. R. Sanes (1987) Topographically selective reinnervation of adult mammalian muscles. Soc. Neurosci. Abstr. 13: 1422.

Lichtman, J. W., and D. Purves (1980) The elimination of redundant preganglionic innervation to hamster sympathetic ganglion cells in early post-natal life. J. Physiol. (Lond.) 301: 213-228.

Magchielse, T., and E. Meeter (1986) The effect of neuronal activity on the competitive elimination of neuromuscular junctions in tissue culture. Dev. Brain Res. 25: 211-220.

Meyer, R. L. (1983) Tetrodotoxin inhibits the formation of refined retinotopography in goldfish. Dev. Brain Res. 6: 293-298.

Miyata, Y., and K. Yoshiuka (1980) Selective elimination of motor nerve terminals in the rat soleus muscle during development. J. Physiol. (Lond.) 309: 631-646.

Navarrete, R., and $G$. Vrbova (1983) Changes of activity patterns in slow and fast muscles during postnatal development. Dev. Brain Res. 8: $11-19$.

O'Brien, R. A. D., J. C. Ostberg, and G. Vrbova (1978) Observations on the elimination of polyneuronal innervation in developing mammalian skeletal muscle. J. Physiol. (Lond.) 282: 571-582.

O'Brien, R. A. D., J. C. Ostberg, and G. Vrbova (1984) Protease inhibitors reduce the loss of nerve terminals induced by activity and calcium in developing rat soleus muscles in vitro. Neuroscience 12: 637-646.

Pestronk, A., and D. B. Drachman (1978) A new stain for quantitative measurement of sprouting at neuromuscular junctions. Muscle Nerve 1: 70-74.

Redfern, P. A. (1970) Neuromuscular transmission in new-born rats. J. Physiol. (Lond.) 209: 701-709.

Reh, T. A., and M. Constantine-Paton (1985) Eye-specific segregation requires neural activity in three eyed Rana pipiens. J. Neurosci. 5: 1132-1143.

Reiter, H. O., and M. P. Stryker (1988) Neural plasticity without postsynaptic action potentials: Less-active inputs become dominant when kitten visual cortical cells are pharmacologically inhibited. Proc. Natl. Acad. Sci. USA 85: 3623-3627. 
Ribchester, R. R. (1988a) Competitive elimination of neuromuscular synapses. Nature 331: 21.

Ribchester, R. R. (1988b) Activity-dependent and -independent synaptic interactions during reinnervation of partially denervated rat muscle. J. Physiol. (Lond.) 401: 53-75.

Ribchester, R. R., and T. Taxt (1983) Motor unit size and synaptic competition in rat lumbrical muscles reinnervated by active and inactive motor axons. J. Physiol. (Lond.) 344: 89-111.

Ribchester, R. R., and T. Taxt (1984) Repression of inactive motor nerve terminals in partially denervated rat muscle after regeneration of active motor axons. J. Physiol. (Lond.) 347: 497-511.

Ridge, R. M. A. P., and W. J. Betz (1984) The effect of selective, chronic stimulation on motor unit size in developing rat muscle. $\mathrm{J}$, Neurosci, 4: 2614-2620.

Singer, W. (1987) Activity dependent self-organization of synaptic connections as a substrate of learning. In The Neural Molecular Bases of Learning, J.-P. Changeux and M. Konishi, eds., pp. 30I-336, Wiley, Chichester, UK.

Smalheiser, N. R., and S. M. Crain (1984) The possible role of "sibling neurite bias" in the coordination of neurite extension, branching, and survival. J. Neurobiol. 15: 517-529.

Soha, J. M., C. Yo, and D. C. Van Essen (1987) Synapse elimination by fiber type and maturational state in rabbit soleus muscle. Dev. Biol. 123: 136-144.

Soha, J. M., E. M. Callaway, and D. C. Van Essen (1989) Lack of fiber type selectivity during reinnervation of neonatal rabbit soleus muscle. Dev. Biol. 131: 401-414.
Thompson, W. J. (1983a) Lack of segmental selectivity in elimination of synapses from soleus muscle of new-born rats. J. Physiol. (Lond.) 335: 343-352.

Thompson, W. (1983b) Synapse elimination in neonatal rat muscle is sensitive to pattern of muscle use. Nature 302:614-616.

Thompson, W. J. (1985) Activity and synapse elimination at the neuromuscular junction. Cell. Mole. Neurobiol. 5: 167-182.

Thompson, W., and J. K. S. Jansen (1977) The extent of sprouting of remaining motor units in partly denervated immature and adult rat soleus muscle. Neuroscience $2: 523-535$.

Thompson, W., D. P. Kuffler, and J. K. S. Jansen (1979) The effect of prolonged, reversible block of nerve impulses on the elimination of polyneuronal innervation of new-born rat skeletal muscle fibers. Neuroscience 4: 271-281.

Thompson, W. J., L. A. Sutton, and D. A. Riley (1984) Fiber type composition of single motor units during synapse elimination in neonatal rat soleus muscle. Nature 309: 709-711.

Van Essen, D. C. (1982) Neuromuscular synapse elimination. In Neuronal Development, N. C. Spitzer, ed., pp. 333-376, Plenum, London.

Wigston, D. J., and J. R. Sanes (1982) Selective reinnervation of adult mammalian muscle by axons from different segmental levels. Nature 299: 464-467.

Wigston, D. J., and J. R. Sanes (1985) Selective reinnervation of intercostal muscles transplanted from different segmental levels to a common site. J. Neurosci. 5: 1208-1221. 